\title{
Local Electron-Lattice Interactions in High-Temperature Cuprate Superconductors
}

\author{
Hugo Keller' ${ }^{1}$ and Annette Bussmann-Holder ${ }^{2}$ \\ ${ }^{1}$ Physik-Institut Universität Zürich, Winterthurerstraße 190, 8057 Zürich, Switzerland \\ ${ }^{2}$ Max-Planck-Institut für Festkörperforschung, Heisenbergstraße 1, 70569 Stuttgart, Germany \\ Correspondence should be addressed to Annette Bussmann-Holder, a.bussmann-holder@fkf.mpg.de
}

Received 26 June 2009; Accepted 29 July 2009

Academic Editor: Sasha Alexandrov

Copyright (C) 2010 H. Keller and A. Bussmann-Holder. This is an open access article distributed under the Creative Commons Attribution License, which permits unrestricted use, distribution, and reproduction in any medium, provided the original work is properly cited.

\begin{abstract}
Recent experimental observations of unconventional isotope effects, multiband superconductivity, and unusual local lattice responses are reviewed and shown to be naturally explained within a two-component scenario where local polaronic effects are important. It is concluded that purely electronic mechanisms of high-temperature superconductivity are incomplete and unable to capture the essential physics of cuprates and other layered superconductors.
\end{abstract}

\section{Introduction}

The discovery of high-temperature superconductivity (HTSC) in cuprates [1] was motivated by the knowledge that copper is one of the strongest Jahn-Teller ions in the periodic system [2]. The basic ingredient of the Jahn-Teller effect is the lifting of the electronic band degeneracy due to a lattice distortion. This has been shown to give rise to Jahn-Teller polaron formation [3] where electronic and lattice degrees of freedom are undistinguishable and form a new quasiparticle sharing the same wave function. Thus lattice and electronic responses are coupled and give rise to multiple novel effects which are absent in a conventional Fermi liquid and an electron-phonon coupled superconductor.

However, soon after the discovery of HTSC, novel mechanisms have been suggested as origin of the pairing glue which neglect completely any lattice responses. These ideas have mainly been invoked in view of the facts that (i) at optimum doping, that is, at the maximum value of the superconducting transition temperature $T_{c}$, the isotope effect on $T_{c}$ is negligibly small [4]; (ii) the superconducting transition temperatures above liquid nitrogen are beyond the BCS scheme; (iii) the antiferromagnetic (AFM) properties of the undoped insulating parent compounds are a consequence of a strong onsite Coulomb repulsion; (iv) a $d$ wave superconducting order parameter seems to be realized.
These facts have been taken as evidence that $t-J$ physics or a two-dimensional Hubbard model is sufficient to capture the essential ingredients of the physics of cuprate hightemperature superconductors (HTSs).

That these ideas are insufficient in explaining the phenomenon of HTSC in cuprates has been demonstrated by the observation of strong lattice responses at the onset of the pseudogap phase as well as at $T_{c}$ [5-12]. Phonon anomalies and anomalous local lattice responses have been reported for both regimes [13-15]. Unexplained within the above mentioned electronic models are all isotope effects observed in the cuprates. These range from a doping dependent isotope effect on $T_{c}[4,16]$, the superconducting energy gap [17], the penetration depth [16], the Néel temperature $T_{N}$ [18], the spin glass phase temperature $T_{g}[18]$, and the pseudogap onset temperature $T^{*}$ [19]. This implies that over the whole and very complex phase diagram of cuprates isotope effects are observed which will be discussed in more detail below. Even though these findings do not necessarily support a lattice mediated pairing mechanism, they are evidence that lattice effects play an essential role and cannot be neglected in any models for HTSC.

Another support for the importance of the lattice to HTSC stems from the fact that superconductivity is only observed upon doping the stoichiometric parent compounds which leads to an energy imbalance in all important physical 
properties: the extra charge introduced by doping leads to a charge mismatch which has to be compensated; the size difference of the doped ions creates local strain fields surrounding the dopant; the antiferromagnetism is rapidly destroyed; in spite of the large onsite Coulomb repulsion the system adopts metallic properties.

The failure of purely electronic models to describe the physics of cuprate HTSs is also demonstrated by the observation that superconductivity is not based on a purely $d$-wave order parameter, but that at least two components are involved here. This has been postulated early on [20, 21] and shown unambiguously recently [22-25]. Details are elucidated below.

The local lattice response to superconductivity as well as to the pseudogap phase has been shown to be quite unusual since the mean square $\mathrm{Cu}-\mathrm{O}$ displacement exhibits novel features at each of these phases [7, 8, 26-28]. The normally expected Debye-Waller behavior appears as a background here, and divergences in it at $T^{*}$ and $T_{c}$ are overlaid on it. These features are rather uncommon in solid state physics and certainly beyond any purely electronic model. They are not observed in a specific cuprate family only, but in all investigated compounds from which it can be concluded that they are intrinsic and generic.

As has been demonstrated early, cuprates are highly inhomogeneous with multiple components which interact with each other but are governed by different-even though coexisting-ground states [20-25, 29-33]. The energy imbalance introduced by dopants has been explained in terms of polaron formation [34] where a transition from a polaron liquid to a polaron glass has been suggested to be realized $[35,36]$. Another approach where lattice effects are of vital importance is based on bipolaron formation where preformed pairs Bose condense at $T_{c}$ [37]. Rather related is the idea that superconducting islands form above $T_{c}$ which form percolating path ways and gain coherence at $T_{c}$ [38-40]. The two-component scenario is also an important ingredient in a two-story house model where anti-Jahn-Teller physics attains importance [41]. Another approach is based on the idea that a crossover from a Bose Einstein to BCS scenario takes place $[29,30]$. Not all of the above ideas are able to explain the unconventional isotope effects, but especially models including polaron/bipolaron formation [42] show that these are realized in agreement with experimental observations [37, 43-46]. The suggestion of multiband superconductivity in complex materials has been made soon after the BCS theory [47-50], and it has been reinvoked for cuprate HTSs after their discovery [5154]. Thus it seems that many experiments and also many theoretical approaches clearly abandon the plain vanilla idea and support each other in a very consistent way.

In the following first experimental results are presented. In the beginning isotope experiments are described, then data presented which strongly support multiband superconductivity in cuprate HTSs, followed by the description of experiments on local lattice responses. Next, a multiband model with polaronic coupling is introduced which consistently describes the experimental data. Finally, conclusions are made.

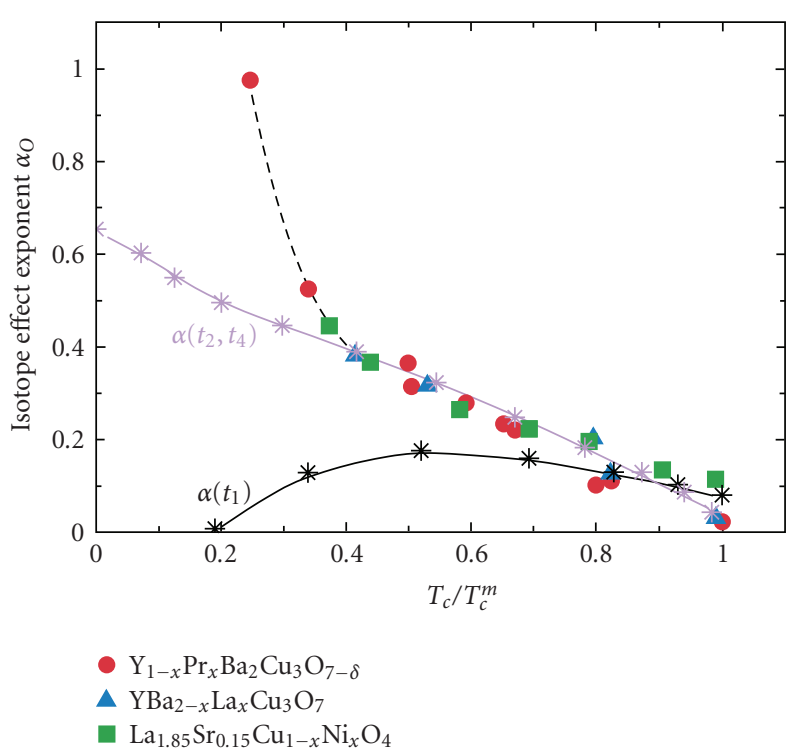

Figure 1: Oxygen isotope $\left({ }^{16} \mathrm{O} /{ }^{18} \mathrm{O}\right)$ effect (OIE) exponent $\alpha_{O}$ versus $t_{c}=T_{c} / T_{c}^{m}$ for various families of cuprate HTSs ( $T_{c}^{m}$ denotes the maximum $T_{c}$ for a particular family). Red circles: $\mathrm{Y}_{1-x} \mathrm{Pr}_{x} \mathrm{Ba}_{2} \mathrm{Cu}_{3} \mathrm{O}_{7-\delta}$; blue triangles: $\mathrm{YBa}_{2-x} \mathrm{La}_{x} \mathrm{Cu}_{3} \mathrm{O}_{7}$; green squares: $\mathrm{La}_{1.85} \mathrm{Sr}_{0.15} \mathrm{Cu}_{1-x} \mathrm{Ni}_{x} \mathrm{O}_{4}$. The references to the experimental data are given in [16]. The black dashed line is a guide to the eye. The black stars and solid line refer to the calculated $\alpha_{O}$ when only the nearest neighbour hopping integral $t_{1}$ is renormalized, whereas the purple stars and solid line refer to the renormalization of $t_{2}, t_{4}$ [43-46].

\section{Experimental Results}

2.1. Isotope Effects. The oxygen-isotope $\left({ }^{16} \mathrm{O} /{ }^{18} \mathrm{O}\right)$ effect (OIE) on the transition temperature $T_{c}$ in HTSs was investgated already shortly after the discovery of HTSC [4]. The OIE on $T_{c}$ is defined by the OIE exponent $\alpha_{O}=$ $-d \ln T_{c} / d \ln M_{O}$, where $M_{O}$ denotes the oxygen ion mass $\left({ }^{16} \mathrm{O}\right.$ or $\left.{ }^{18} \mathrm{O}\right)$. Numerous experiments revealed that for all cuprate HTS families the OIE exponent $\alpha_{O}$ shows a generic trend: in the underdoped regime $\alpha_{O}$ is large (even exceeding the BCS value of $\alpha_{O}=0.5$ ) and becomes small close to optimal doping $[4,16,56,57]$. An example of this generic behavior of $\alpha_{O}$ as a function of $T_{c} / T_{c}^{m}$ for various families of cuprate HTSs is displayed in Figure 1 ( $T_{c}^{m}$ denotes the maximum $T_{c}$ for a particular HTS family).

The almost vanishing OIE at optimum doping is contrasted to the strongly enhanced one in the underdoped limit close to the antiferromagnetic (AFM) phase boundary. Here, the OIE well exceeds the BCS limit and exhibits unusually large values. This is striking since the proximity to the AFM regime would suggest that spin fluctuations gain importance and dominate over any lattice effects. This observation clearly marks the breakdown of purely electronic models.

The early suggestion that the apex oxygen ions contribute in a special way to superconductivity stems from the fact that strongly anharmonic dynamics are involved in out of plane oxygen ion vibrations [58-62]. These polar almost instable modes carry huge dipole moments and cause the 


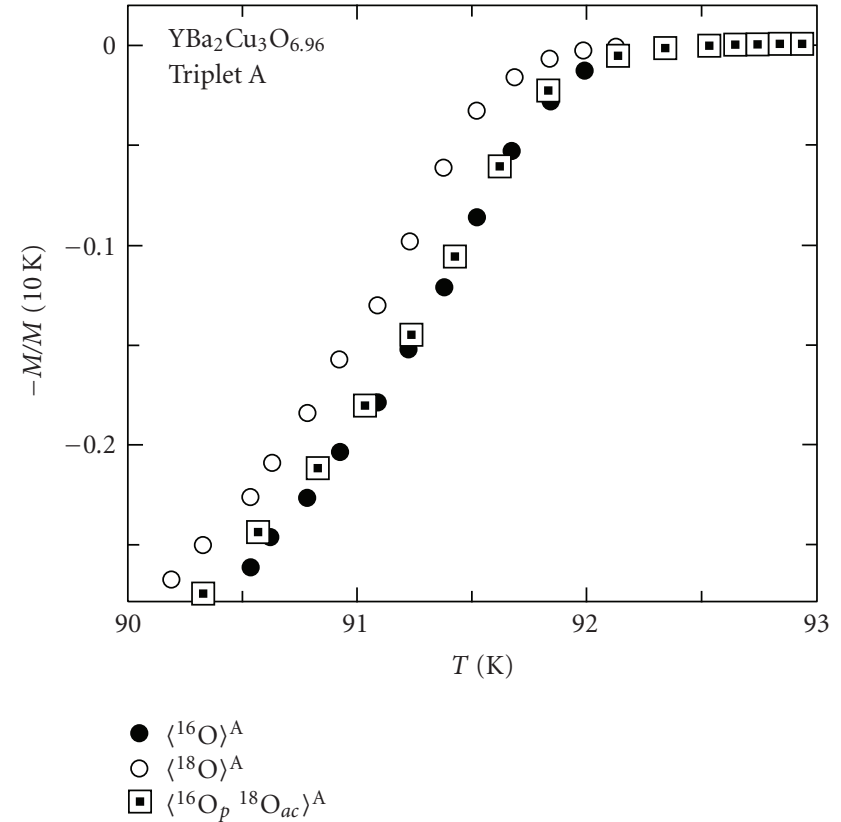

(a)

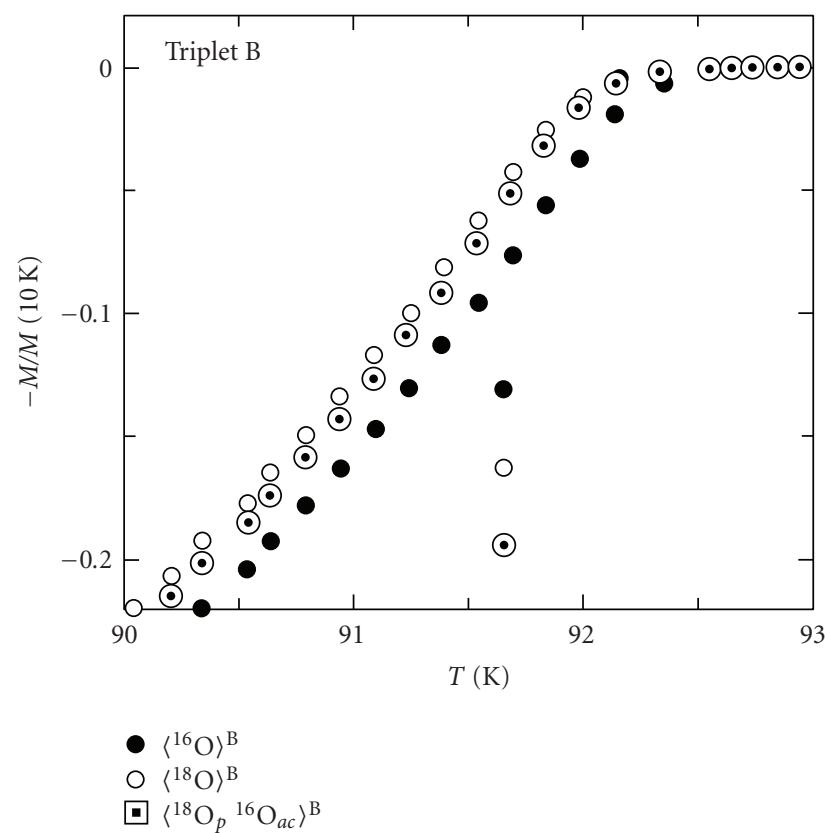

(b)

Figure 2: Magnetization curves near $T_{c}$, showing the SOIE in optimally doped $\mathrm{YBa}_{2} \mathrm{Cu}_{3} \mathrm{O}_{7-\delta}$ for $x=6.957(2)$ (triplet A) and $x=6.963(3)$ (triplet $\mathrm{B}$ ). Here $p, a$, and $c$ denote the different oxygen ion sites in $\mathrm{YBa}_{2} \mathrm{Cu}_{3} \mathrm{O}_{7-\delta}$ ( $p$ : planar oxygen ion sites; $a$ : apical oxygen ion sites; $c$ : chain oxygen ion sites). For example, $\left\langle{ }^{18} \mathrm{O}_{p}{ }^{16} \mathrm{O}_{a c}\right\rangle{ }^{\mathrm{B}}$ denotes a sample from batch $\mathrm{B}$ with ${ }^{18} \mathrm{O}$ in $\mathrm{CuO}_{2}$ planes and ${ }^{16} \mathrm{O}$ in the apical and chain sites. It is evident that the planar oxygen atoms mainly contribute to the total OIE on $T_{c}$, after [55].

high dielectric constants observed in cuprate HTSs [63]. In order to test these ideas, site selective oxygen-isotope effect (SOIE) experiments are the only tool to differentiate between the role played by the in-plane and the out of plane oxygen ions. SOIE experiments are extremely difficult, and so far could only be realized for the $\mathrm{Y}_{1-x} \mathrm{Pr}_{x} \mathrm{Ba}_{2} \mathrm{Cu}_{3} \mathrm{O}_{7-\delta}$ system, since for this system the oxygen ion site occupation can be controlled thermally [55, 64-66]. Careful back exchange experiments were performed to ensure that the doping level for both isotopes remains identical. In addition, Raman experiments were undertaken to clearly differentiate from the eigenmodes where oxygen isotope replacements were made. As an example, Figure 2 shows the magnetization curves near $T_{c}$ of the SOIE study of optimally doped $\mathrm{YBa}_{2} \mathrm{Cu}_{3} \mathrm{O}_{7-\delta}$ by Zech et al. [55]. It is evident that the planar oxygen ions mainly contribute to the total OIE on $T_{c}$. Moreover, detailed SOIE investigations of $\mathrm{Y}_{1-x} \mathrm{Pr}_{x} \mathrm{Ba}_{2} \mathrm{Cu}_{3} \mathrm{O}_{7-\delta}$ clearly revealed that the planar oxygen atoms mainly contribute (almost $100 \%)$ to the total OIE on $T_{c}$ at all doping levels, whereas the chain and apical oxygen ions contribute only negligibly to it (see Figure 3) [55, 64-66]. This finding could point to a dominant role played by the in-plane oxygen ions, but this is, however, misleading since the density of states of the inplane oxygen ions is much larger than the one of the outof-plane oxygen ions and thus it cannot be concluded that these ions are irrelevant to superconductivity, which will be detailed below [67].

An OIE on the in-plane magnetic penetration depth $\lambda_{a b}$ should be absent within the BCS theory since electronic

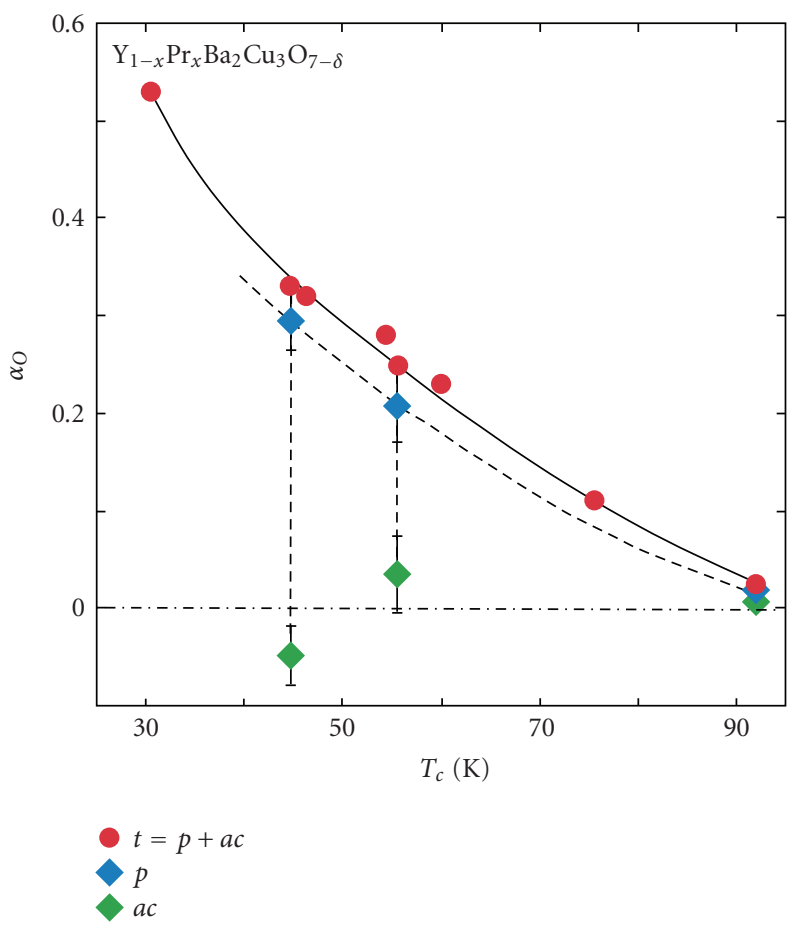

Figure 3: Total $(t)$ and partial $(p, a c)$ OIE exponent $\alpha_{O}$ as a function of $T_{c}$ for $\mathrm{Y}_{1-x} \mathrm{Pr}_{x} \mathrm{Ba}_{2} \mathrm{Cu}_{3} \mathrm{O}_{7-\delta}$ ( $t$ : all oxygen ion sites; $p$ : planar oxygen ion sites; ac: apex and chain oxygen ion sites). Solid and dashed lines are guides to the eye, after [16]. 


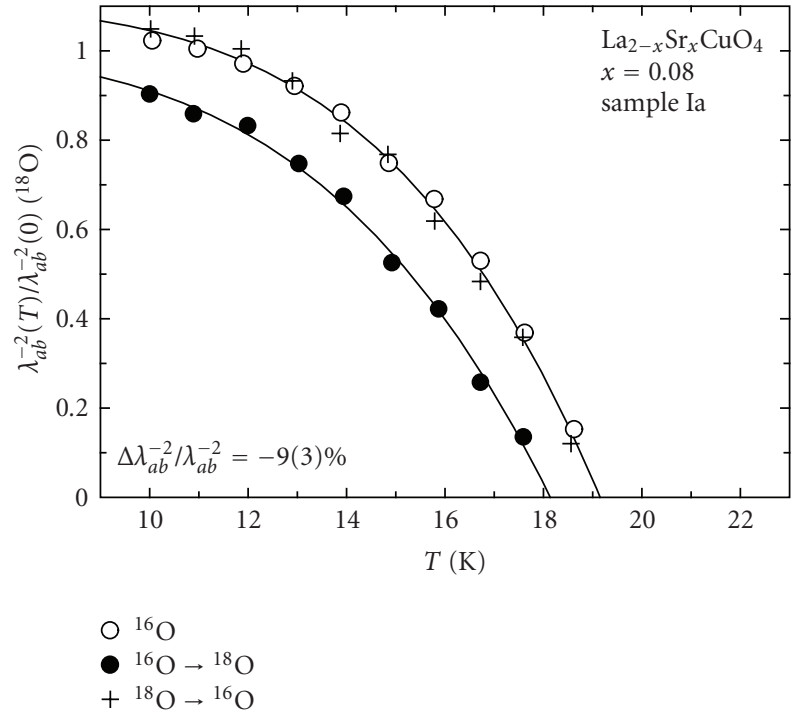

(a)

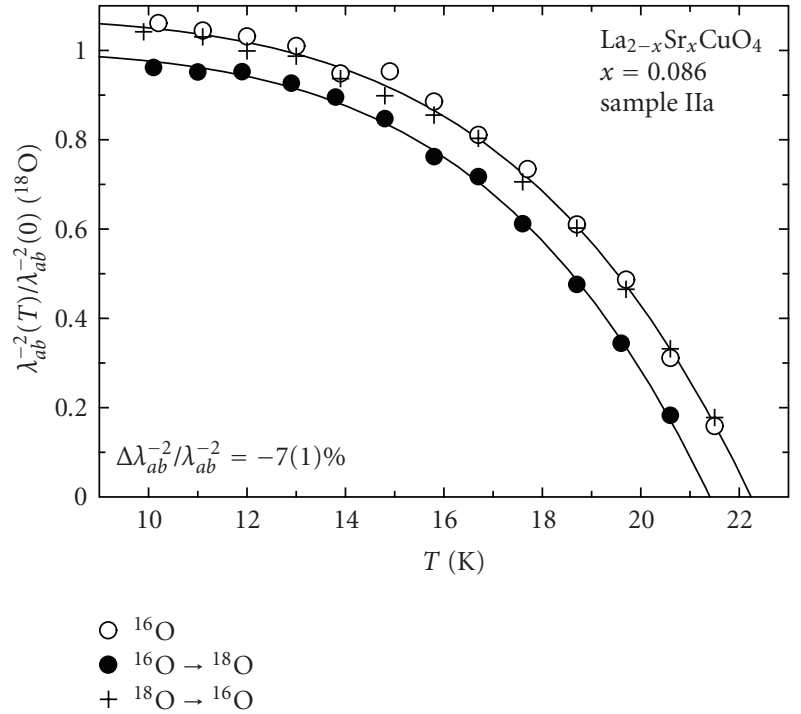

(b)

Figure 4: Normalized superfluid density (in-plane penetration depth) $\lambda_{a b}^{-2}(T) / \lambda_{a b}^{-2}(0)$ for the ${ }^{16} \mathrm{O}$ - and ${ }^{18} \mathrm{O}$-substituted microcrystals of $\mathrm{La}_{2-x} \mathrm{Sr}_{x} \mathrm{CuO}_{4}((\mathrm{a}): x=0.080$, (b): $x=0.086)$. The reproducibility of the oxygen exchange was checked by the back exchange (crosses), after [68].

and phononic degrees of freedom are treated as independent (adiabatic approximation). In the opposite limit, however, an isotope effect on $\lambda_{a b}$ is possible, which corresponds to strong coupling as is given in polaronic models. This implies that the effective carrier mass $m^{*}$ can no longer be decoupled from the ionic mass $M$, giving rise to an isotope effect on the magnetic penetration depth which is not expected for conventional phonon-mediated superconductors. Indeed, a substantial OIE on the zero-temperature in-plane magnetic penetration depth $\lambda_{a b}(0)$ was observed in several families of HTSs at different doping levels using various experimental techniques $[16,56,57,68,70-72]$. As an example, Hofer et al. [68] investigated the OIE on $T_{c}$ and $\lambda_{a b}(0)$ in microcrystals of underdoped $\mathrm{La}_{2-x} \mathrm{Sr}_{x} \mathrm{CuO}_{4}$ with a mass of only $\approx 100 \mu \mathrm{g}$ using a highly sensitive magnetic torque device to measure the magnetization of these tiny crystals. Figure 4 shows the temperature dependence of $\lambda_{a b}^{-2}(T) / \lambda_{a b}^{-2}(0)$ extracted from the magnetic torque data for two oxygen-isotope exchanged microcrystals of $\mathrm{La}_{2-x} \mathrm{Sr}_{x} \mathrm{CuO}_{4}$.

The doping dependent OIE on $\lambda_{a b}(0)$ is appreciable as can be seen in Figure 4. Since it is observed in various different cuprates, it must be concluded that it is generic to HTSs. It is interesting to note that the OIE on $\lambda_{a b}(0)$ found for different families of cuprates exhibits almost the same generic trend with doping as the one on $T_{c}$ (see Figure 5). At low doping a linear correlation between both OIE's is observed: $\Delta \lambda_{a b}(0) / \lambda_{a b}(0) \approx\left|\Delta T_{c} / T_{c}\right|$. However, near optimal doping, a deviation from this linear behaviour occurs, and $\Delta \lambda_{a b}(0) / \lambda_{a b}(0) \approx 10\left|\Delta T_{c} / T_{c}\right|$. The linear correlation at low doping is well explained by the polaronic model [43-46] described below. However, this model cannot account for the deviations near optimum doping. This is due to the fact that the polaronic coupling was assumed to be doping independent which is an oversimplification. At optimum doping the polaronic coupling should be smaller than in the underdoped regime which still would yield an isotope effect on the penetration depth but a vanishing one on $T_{c}$.

The OIE's discussed so far already provide clear evidence that purely electronic models cannot describe the complex physics of HTSs. Another doping dependent OIE on the average zero-temperature superconducting gap $\bar{\Delta}_{0}$ further supports this conclusion.

In conventional superconductors the isotope effect on the superconducting energy gap $\Delta_{0}$ is simply determined by the isotope effect on $T_{c}$ through the relation $2 \Delta_{0} / k_{B} T_{c} \approx$ 3.52. However, in cuprate HTSs this relation is not necessarily fulfilled, because $T_{c}$ depends strongly on doping. In addition, as discussed below, cuprate HTSs have a complex superconducting order parameter, namely, an admixture of $s+d$-wave symmetry. A systematic study of the OIE on the average gap $\bar{\Delta}_{0}=(1 / 2) \sqrt{\bar{\Delta}_{s}^{2}+\bar{\Delta}_{s}^{2}}\left(\bar{\Delta}_{s} \equiv s\right.$-wave gap, $\bar{\Delta}_{d} \equiv d$-wave gap) in $\mathrm{Y}_{1-x} \mathrm{Pr}_{x} \mathrm{Ba}_{2} \mathrm{Cu}_{3} \mathrm{O}_{7-\delta}$ for various doping levels $x$ was carried out by means of SQUID magnetization experiments [17]. Note that the above defined average gap equally weighs the $s$ - and $d$-wave contributions. In reality the gaps could contribute with different weights to the average one depending on the doping level. However, this would introduce an additional parameter which is not accessible experimentally. For this reason we have assumed the simplest possible form of the average gap as given above. The values of $\bar{\Delta}_{0}$ extracted from the temperature dependence of the superfluid density were found to be proportional to $T_{c}$ with $2 \bar{\Delta}_{0} / k_{B} T_{c} \approx 5.34$ as predicted prior to the experiment by the polaron model [43-46] described here. It is substantially larger than the BCS value of 3.52. As shown in Figure 6, the OIE on $\bar{\Delta}_{0}$ scales linearly with the one on $T_{c}$, exhibiting a 


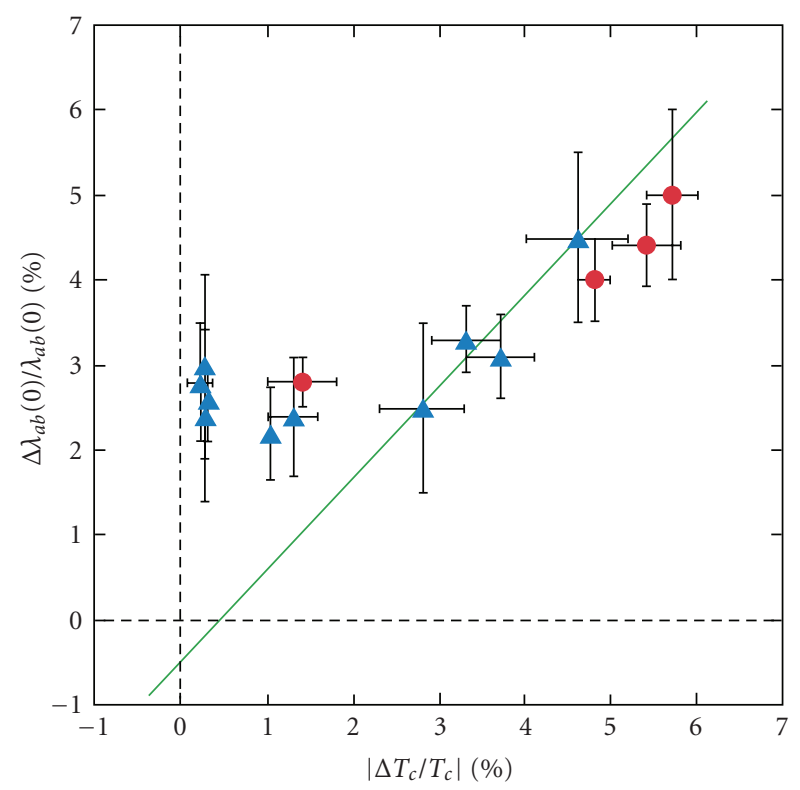

- $\mathrm{La}_{2-x} \mathrm{Sr}_{x} \mathrm{CuO}_{4}$

$\Delta \mathrm{Y}_{1-x} \mathrm{Pr}_{x} \mathrm{Ba}_{2} \mathrm{Cu}_{3} \mathrm{O}_{7-\delta}$

Figure 5: Plot of the OIE shift $\Delta \lambda_{a b}(0) / \lambda_{a b}(0)$ versus the OIE shift $\left|\Delta T_{c} / T_{c}\right|$ for $\mathrm{La}_{2-\chi} \mathrm{Sr}_{x} \mathrm{CuO}_{4}$ and $\mathrm{Pr}_{x} \mathrm{Y}_{1-x} \mathrm{Ba}_{2} \mathrm{Cu}_{3} \mathrm{O}_{7-\delta}$ using different experimental techniques and types of samples. The data are summarized in [16, Table 1]. The solid line corresponds to model calculations of the OIE on $\Delta \lambda_{a b}(0) / \lambda_{a b}(0)$ obtained from the theoretical results for the average gap [43-46, 69], after [43-46].

sign reversal of the OIE's on $\bar{\Delta}_{0}$ and $T_{c}$ near optimal doping as predicted in [43-46] and discussed below.

Cuprate HTSs exhibit a rich phase diagram as a function of doping (see Figure 7). The undoped parent compounds show long range $3 \mathrm{D}$ antiferromagnetic (AFM) order. When holes are doped into the $\mathrm{CuO}_{2}$ planes, the AFM order is rapidly destroyed and only short-range AFM correlations survive even in the superconducting (SC) region of the phase diagram giving rise to a spin-glass (SG) state. Consequently, the SC and the SG phases coexist within a small doping range. With increasing doping, the SG phase disappears and a pure SC phase with increasing superconducting transition temperature $T_{c}$ emerges. Four different phases can thus be distinguished: the AFM phase, the SG phase, the SG + SC phase, and the SC phase. The relation and interplay of these phases is still a controversial and open issue, since key experiments that may clarify this fundamental questions are still missing. Therefore, it is very interesting to investigate the OIE's on the corresponding transition temperatures between the various phases.

Several years ago the Zurich group observed a huge OIE on the spin-glass freezing temperature $T_{g}$ in $\mathrm{La}_{2-x} \mathrm{Sr}_{x} \mathrm{Cu}_{1-z} \mathrm{Mn}_{z} \mathrm{O}_{4}(x=0.03$ and $0.05 ; z=0.02)$ by means of muon-spin rotation $(\mu \mathrm{SR})$ [73]. This is a clear signature that the spin dynamics in cuprates are ultimately correlated with the lattice. Recently, the same group performed a detailed OIE study of the various phases (SC, SG + SC, SG, AFM) in the prototype cuprate system

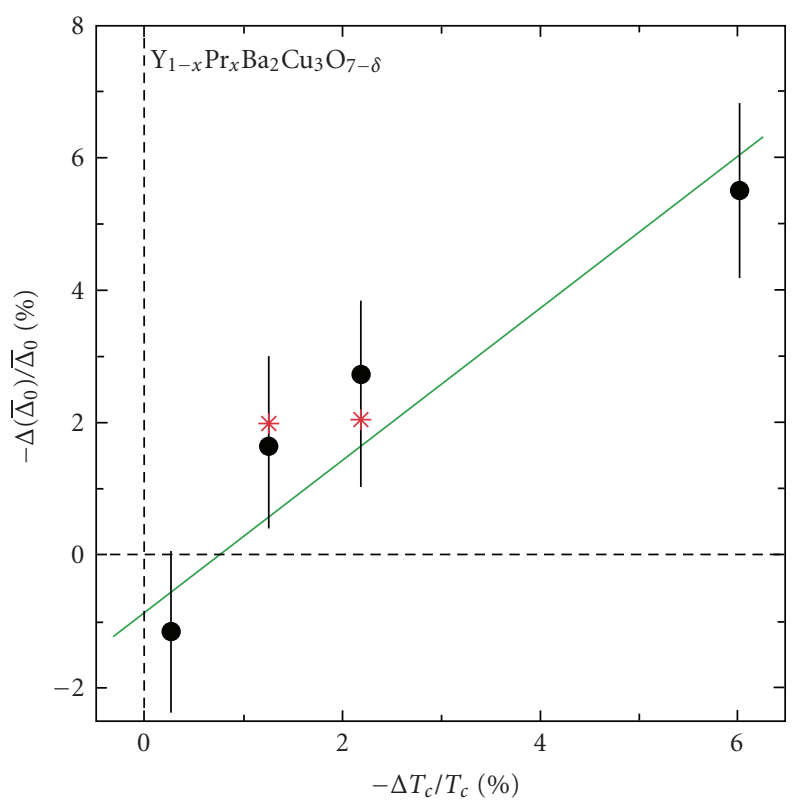

FIgure 6: Comparison of the OIE shift of the average superconducting gap $\bar{\Delta}_{0}$ to the one on the transition temperature $T_{c}$ for $\mathrm{Y}_{1-x} \mathrm{Pr}_{x} \mathrm{Ba}_{2} \mathrm{Cu}_{3} \mathrm{O}_{7-\delta}(x=0.0,0.2,0.3,0.45)$. Circles refer to the experimental data. The stars represent back exchange data. The solid green line is discussed, after [17].

$\mathrm{Y}_{1-x} \mathrm{Pr}_{x} \mathrm{Ba}_{2} \mathrm{Cu}_{3} \mathrm{O}_{7-\delta}$ by means of $\mu \mathrm{SR}$ and magnetization experiments [18]. These techniques have the advantage of being direct, bulk sensitive, unambiguous, and able to measure $T_{c}$ as well as $T_{g}$ in the region where both coexist. The results of this OIE study are displayed in Figure 7. All transition temperatures $T_{c}, T_{g}$, and $T_{N}$ exhibit an OIE which is the strongest, where the respective phase (SC, SG, and AFM) terminates. It is interesting to note that the OIE on $T_{g}$ and $T_{N}$ are sign reversed as compared to the one on $T_{c}$. In the coexistence region of the SG and SC phase (SG + SC) a small OIE on $T_{c}$ corresponds to a large negative OIE on $T_{g}$ and vice versa. This observation suggests that in this regime phase separation sets in where the superfluid density coexists with a nonsuperfluid density related to the SG state. Since the OIE on $T_{c}$ can be accounted for by polaron formation [43-46], the one on $T_{g}$ is very likely driven by the same physics. By relating $T_{N}$ to the metal insulator transition, a reduction in kinetic energy caused by polaron formation explains this unconventional OIE as well [18, 43-46].

In addition to the OIE's observed on all the phase lines in the generic phase diagram of cuprate HTSs (see Figure 7(a)), a huge OIE was also detected on the pseudogap temperature $T^{*}$, providing further evidence that lattice effects are relevant here as well. In particular, XANES experiments in $\mathrm{La}_{2-x} \mathrm{Sr}_{x} \mathrm{CuO}_{4}$ [74] as well as inelastic neutron scattering studies of $\mathrm{HoBa}_{2} \mathrm{Cu}_{4} \mathrm{O}_{8}$ and $\mathrm{La}_{1.81} \mathrm{Sr}_{0.18} \mathrm{Ho}_{0.04} \mathrm{CuO}_{4}$ [19] yielded a large and sign reversed OIE on $T^{*}$. As an example, the results for $\mathrm{HoBa}_{2} \mathrm{Cu}_{4} \mathrm{O}_{8}$ are shown in Figure 8. It is interesting to note that neutron scattering experiments revealed an even larger ${ }^{63} \mathrm{Cu} /{ }^{65} \mathrm{Cu}$ isotope effect on $T^{*}$ which is not present in $\mathrm{La}_{1.81} \mathrm{Sr}_{0.18} \mathrm{Ho}_{0.04} \mathrm{CuO}_{4}$ [19]. This finding 


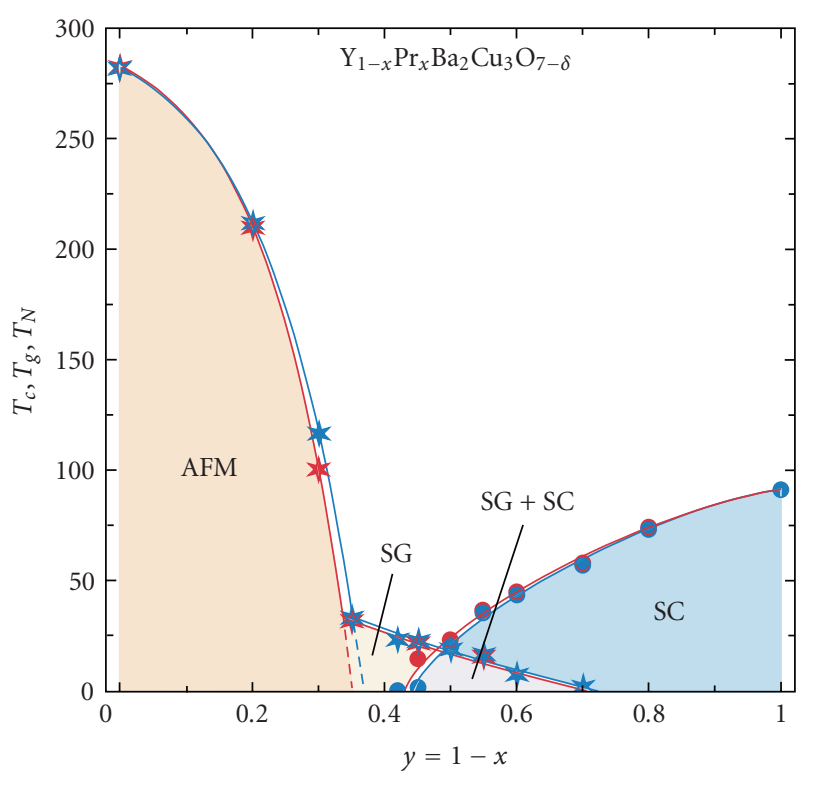

ㅈ ${ }^{16} \mathrm{O}$
$\times \times{ }^{18} \mathrm{O}$

(a)

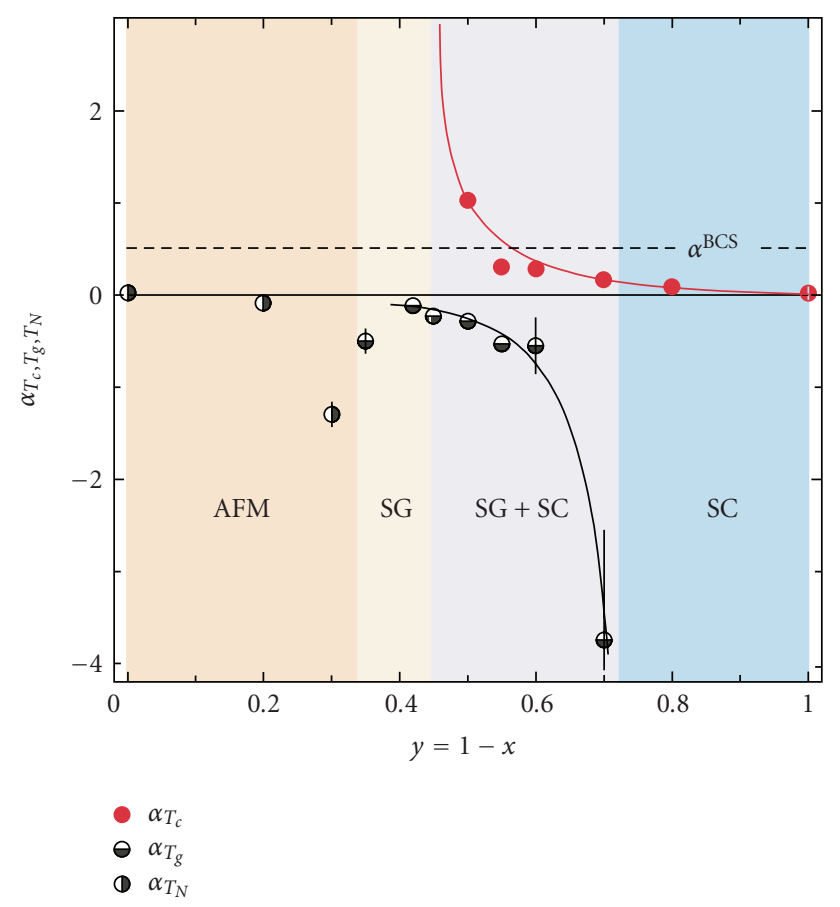

(b)

Figure 7: (a) Dependence of the superconducting transition $\left(T_{c}\right)$, the spin-glass ordering $\left(T_{g}\right)$, and the antiferromagnetic ordering $\left(T_{N}\right)$ temperatures for ${ }^{16} \mathrm{O} /{ }^{18} \mathrm{O}$-substituted $\mathrm{Y}_{1-x} \mathrm{Pr}_{x} \mathrm{Ba}_{2} \mathrm{Cu}_{3} \mathrm{O}_{7-\delta}$ on the $\operatorname{Pr}$ content $y=1-x$. The solid lines are guides to the eye. The areas-denoted by "AFM", "SG", and "SC" represent the antiferromagnetic, the spin-glass, and the superconducting regions, respectively, and "SG + SC" corresponds to the region where spin-glass magnetism coexists with superconductivity. (b) OIE exponents $\alpha_{O}$ of $T_{c}, T_{g}$, and $T_{N}$ for ${ }^{16} \mathrm{O} /{ }^{18} \mathrm{O}$-substituted $\mathrm{Y}_{1-x} \mathrm{Pr}_{x} \mathrm{Ba}_{2} \mathrm{Cu}_{3} \mathrm{O}_{7}$ as a function of the Pr content $y=1-x$. The dashed line corresponds to the BCS value $\alpha_{O}^{\mathrm{BCS}}=0.5$. The solid lines are guides to the eye, after [18]. supports the idea that an umbrella-type mode is involved in the formation of the pseudogap state in $\mathrm{HoBa}_{2} \mathrm{Cu}_{4} \mathrm{O}_{8}$. Since $\mathrm{La}_{1.81} \mathrm{Sr}_{0.18} \mathrm{Ho}_{0.04} \mathrm{CuO}_{4}$ has no apical oxygen, this mode is absent in this compound. These huge isotope effects have been interpreted in terms of dynamical charge ordering [4346]. The two-component picture discussed in this paper implies that also ordering in the spin system (charge poor region) sets in around $T^{*}$ as observed in $\mathrm{YBa}_{2} \mathrm{Cu}_{4} \mathrm{O}_{8}$ by means of NQR [75]. This is consistent with the almost vanishing OIE on $T^{*}$ (comparable to the OIE on $T_{c}$ ) detected by NQR which is mainly sensitive to the spin system [75].

2.2. Mixed Order Parameter Symmetries. From, for example, phase sensitive experiments [76] it has been concluded that the order parameter in cuprate HTSs has pure $d$-wave symmetry, which is possible only if the $\mathrm{CuO}_{2}$ planes have strictly cubic symmetry. However, either static or dynamic distortions of the $\mathrm{CuO}_{2}$ planes are present which destroy the cubic symmetry and are mostly ignored theoretically. Early on it has been emphasized that cuprate HTSs must have a multicomponent order parameter, since many experiments are incompatible with a single $d$-wave scenario $[20,21]$. This implies that coexisting superconducting gaps with different pairing symmetries must be present, namely, $s$-wave and $d$ wave. This suggestion has been supported by a number of experiments using various experimental techniques, including nuclear magnetic resonance (NMR) [77, 78], Raman scattering [79, 80], phase-sensitive experiments [81], and neutron crystal-field spectroscopy [82], to give only a few examples. In addition, $c$-axis tunneling data provide evidence that the gap along the $c$-axis is dominantly of $s$-wave symmetry $[83,84]$. Soon after the BCS theory coupled gaps have been postulated to be realized in complex materials where various electronic bands lie in the vicinity of the Fermi surface [47-50]. The experimental verification of these ideas was, however, made much later in $\mathrm{Nb}$ doped $\mathrm{SrTiO}_{3}$ [85] and long been believed to be an unusual exception. After the discovery of two-gap superconductivity in $\mathrm{MgB}_{2}$ [86] a vast amount of compounds have been shown to exhibit this phenomenon. Especially, in the newly discovered FeAs superconductors with rather high $T_{c}$ 's unique features for the existence of two gaps have been seen [87]. Since $c$ axis experiments are rare for cuprates and most tunneling data are in the $a b$-plane, different tools have to be used to demonstrate the existence of $s+d$-wave superconductivity in cuprates. In order to probe the existence of coupled order parameters bulk sensitive experiments on single crystals are required. Muon-spin rotation $(\mu \mathrm{SR})$ has demonstrated to be a unique tool to investigate the temperature dependence of the superfluid density in the bulk of a superconductor, from which, in turn, the superconducting gaps can be derived. A clear indication for the existence of two gaps is the appearance of an inflection point in the superfluid density at low temperatures. Such an anomaly was detected in $\mathrm{MgB}_{2}$ and shown to stem from the coexistence of a large and a small gap [86]. In cuprate HTSs a similar anomaly was observed in the in-plane superfluid density in single-crystal $\mathrm{La}_{2} \mathrm{Sr}_{2-x} \mathrm{CuO}_{4}$ determined by means of 


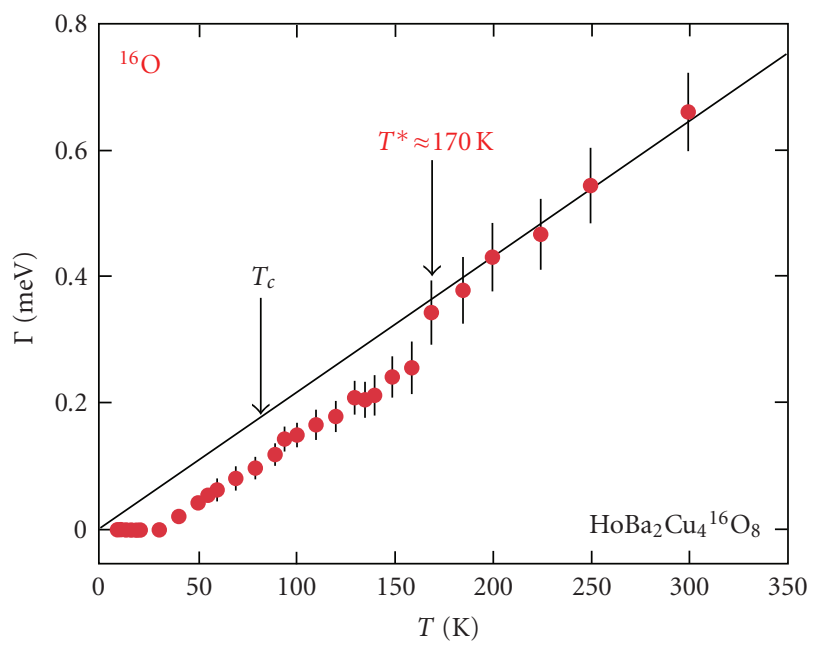

(a)

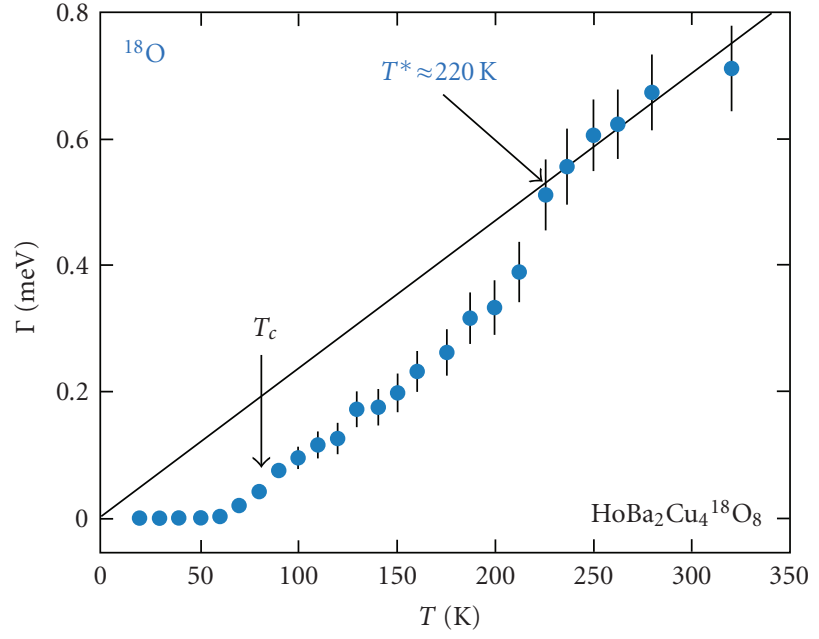

(b)

FIgURE 8: Temperature dependence of the intrinsic line width $\Gamma(\mathrm{HWHM})$ corresponding to the $\Gamma_{3} \rightarrow \Gamma_{4}$ ground-state crystal field transition in oxygen-isotope exchanged $\mathrm{HoBa}_{2} \mathrm{Cu}_{4} \mathrm{O}_{8}$ as determined from inelastic neutron scattering experiments. The solid lines correspond to the line width in the normal state calculated by the Korringa law, after [19].

$\mu \mathrm{SR}$ experiments and analyzed in terms of coupled $s+d$ wave order parameters (Figure 9) [22-25]. In order to show that this feature is not material dependent but intrinsic to cuprates similar experiments were performed for single crystals of $\mathrm{YBa}_{2} \mathrm{Cu}_{3} \mathrm{O}_{7-\delta}$ and $\mathrm{YBa}_{2} \mathrm{Cu}_{4} \mathrm{O}_{8}$ where it was possible to determine the temperature dependence of the superfluid density along all three crystallographic axis $a, b$, and $c$ [22-25]. The results are shown in Figure 10, from which it can be seen that in both compounds an inflection point appears along the $a$ - and $b$-axis, which is absent along the $c$-axis. The analysis of these data yields $s+d$ wave order parameters in the $a b$-planes, whereas along the $c$-axis predominantly an $s$-wave component exists.

The observation of a pronounced inflection point in the in-plane superfluid density at low temperatures in all three systems (Figures 9 and 10) is a signature of the coexistence of a small and a large gap. From the magnetic field dependence of the superfluid density it is concluded [22-25] that in the $\mathrm{CuO}_{2}$ planes a small $s$-wave gap coexists with a dominant $d$-wave gap. As shown in Figure 10, the temperature dependence of the superfluid density along the $c$-axis differs considerably from those in the $b$-planes. The absence of an inflection point and the saturation at low temperatures (compare $\sigma_{a}$ and $\sigma_{b}$ with $\sigma_{c}$ in Figure 10) are characteristic for a pure $s$-wave gap. From a theoretical point of view, a mixed order parameter scenario [43-46] requires that also a small $d$-wave admixture should be present. However, the $d$-wave component appears to be too small to be observed experimentally [22-25].

In conclusion, the unique behaviour of the temperature dependence of the superfluid density observed in all three cuprate systems strongly suggests that the order parameter is more complex than expected for a single $d$-wave order parameter, and that this complex order parameter is an intrinsic and generic feature of all cuprate HTSs. In particular, the finding that the gap along the $c$-axis has a predominant $s$-wave character demonstrates the importance of the coupling between the $\mathrm{CuO}_{2}$-planes, and that 2D physics concentrating on the $\mathrm{CuO}_{2}$ planes only is rather insufficient and incomplete. It is important to mention that the coexistence of an $s$ - and $d$-wave gap not only is a consequence of the static or dynamic degree of orthorhombicity but also results from the fact that two components dominate the physics of HTSs. These experimental facts are completely neglected in many theoretical models.

2.3. Local Lattice Responses. About 10 years after the discovery of HTSC a number of local probes have been used to study the in-plane and out-of-plane $\mathrm{Cu}-\mathrm{O}$ distances in order to gain information about the local structure in cuprates $[5-8,10-13,26-28]$. Here, especially, the extended X-ray absorption fine structure (EXAFS) technique has proven to be a useful tool. The early experiments [5-8] reported not only two distinctly different $\mathrm{Cu}-\mathrm{O}$ bond lengths in the $\mathrm{Cu}$-apical-oxygen distance but also anomalies in these as a function of temperature. While far above $T_{c}$ the first anomaly was observed and related to $T^{*}$, the second developed in the SC phase. Besides of the $c$-axis-related anomalies, also the in-plane $\mathrm{Cu}-\mathrm{O}$ bond length was shown to be characterized by similar anomalies and two length scales $[7,8]$. The interpretation of the data was made in terms of stripe like modulations of the local structure setting in at $T^{*}$, where undistorted and distorted areas coexist. More recently, high-resolution EXAFS studies have concentrated on the inplane $\mathrm{Cu}-\mathrm{O}$ distance and investigated this in the presence of different dopants [26-28]. In the latter studies an anomalous upturn in the mean square in-plane $\mathrm{Cu}-\mathrm{O}$ distance has been reported with two clear anomalies appearing at $T^{*}$ and below $T_{c}$. Typical results are shown in Figure 11 and in comparison to the theoretical results as inset to Figure 16. Note that 


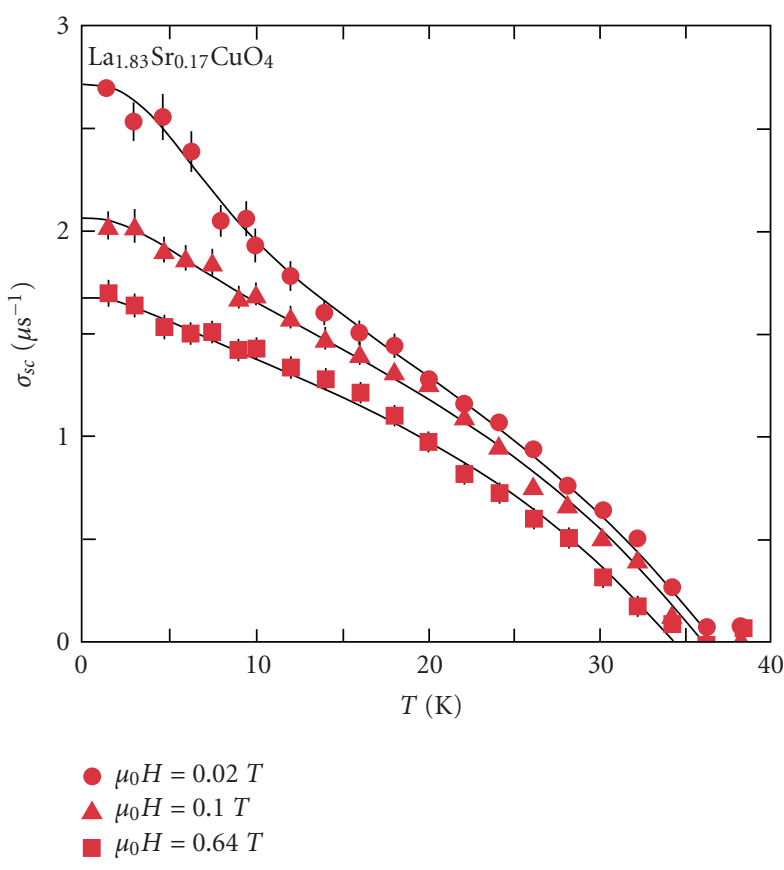

(a)

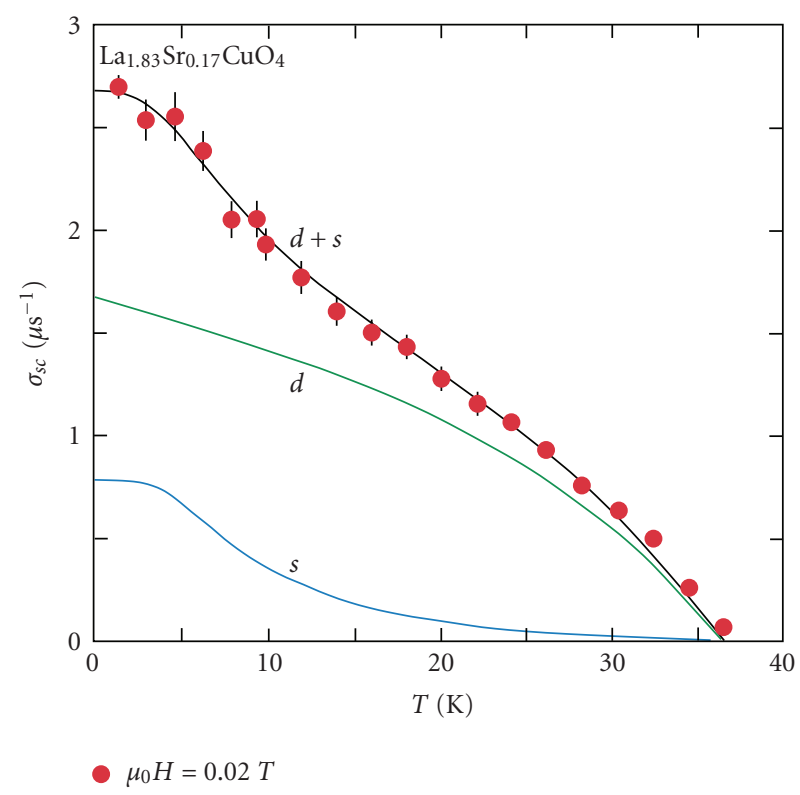

(b)

FIGURE 9: Temperature dependence of the $\mu$ SR relaxation rate $\sigma_{s c} \propto \lambda_{a b}^{-2}$ of single crystals of $\mathrm{La}_{1.83} \mathrm{Sr}_{0.17} \mathrm{CuO}_{4}$ measured at magnetic field strengths of $0.02,0.1$, and $0.64 \mathrm{~T}$. Lines in panels (a) and (b) represent calculations using a two-gap model [22-25]. The green and blue lines in panel (b) show the individual contributions from the $d$ - and $s$-wave gap, after [22-25].

such anomalies are rather untypical in conventional solids and even absent in highly anharmonic compounds with strong soft mode behaviour. Since the experiments test ionic displacements which correlate with $T^{*}$ and $T_{c}$, an explanation of these is beyond any purely electronic model. In addition, the appearance of these anomalies along the $c$ axis points to pronounced $c$-axis involvement, as has been stressed from the penetration depth data.

\section{Theoretical Modeling}

The theoretical understanding of the pairing mechanism in cuprate HTSs remains controversial where basically two distinctly different approaches have been pursued in the last years: one is based on a purely electronic mechanism where the large onsite Coulomb repulsion $U$ at the $\mathrm{Cu}$ site is assumed to play a major role. This approach can either be cast into a 2D Hubbard model or in extreme cases, when $U$ is much larger than the hopping integral, or be modeled by the so-called $t-J$ Hamiltonian. Obviously, all lattice effects are ignored whereby isotope effects, as observed experimentally and being described above, are regarded as unimportant. Nevertheless, an explanation for these is offered by postulating that the hopping integrals depend on the oxygen isotope mass. This implies that the unique energy scale as given by $J=4 t^{2} / U$ becomes isotope dependent. Since $J$ in this approach dominates the AFM regime as well as the SC region, both corresponding transition temperatures, namely, $T_{N}, T_{c}$, should have equal isotope dependencies. This is in contrast to the above described isotope experiments where $T_{N}$ and $T_{c}$ have opposite signs of the OIE. Also, the many correlations between lattice anomalies and superconductivity are missing in these scenarios. 2D Hubbard and $t-J$ models are based on a single $d$-wave order parameter and do not admit for the complex order parameters as observed experimentally. In this respect both approaches rely on a homogeneous picture in contrast to the observed inhomogeneity of HTSC. A rather dramatic failure of these purely electronic approaches is the inability to account for the $c$-axis $s$-wave gap. The third dimension is almost completely ignored and important contributions from the out-of-plane structural elements missing. As such, the completely decoupled $\mathrm{CuO}_{2}$ planes alone should be superconducting, which is again in contrast to experiments.

An alternative approach to HTSC is based on electronlattice interactions, where conventional BCS theory and polaron and bipolaron formation are considered [29-46]. The problem with the standard electron-phonon-coupled superconductivity lies in the fact that the high values of $T_{c}$ require large coupling constants, which, in turn, may easily lead to lattice instabilities. Also, as already mentioned above, an isotope effect on the penetration depth is absent, and a doping dependent OIE on $T_{c}$ is not present. As such, polaronic or bipolaronic scenarios are more likely to be realized in HTSs. This is also favored by the energy mismatch introduced upon doping. The extra charge which enters the stoichiometric antiferromagnetic matrix requires strong screening through the lattice, which undergoes anomalous local distortions around these charges which have been 

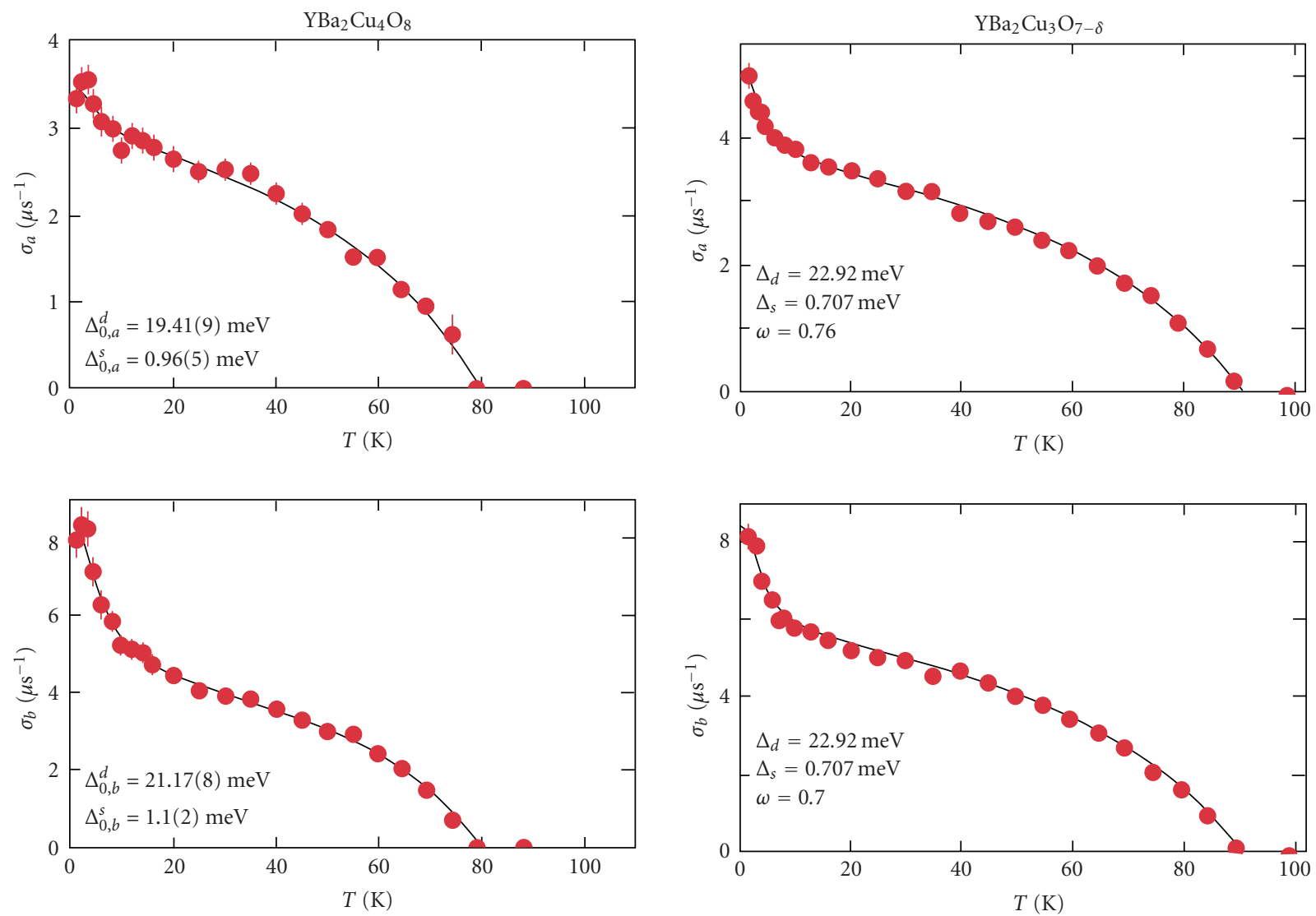

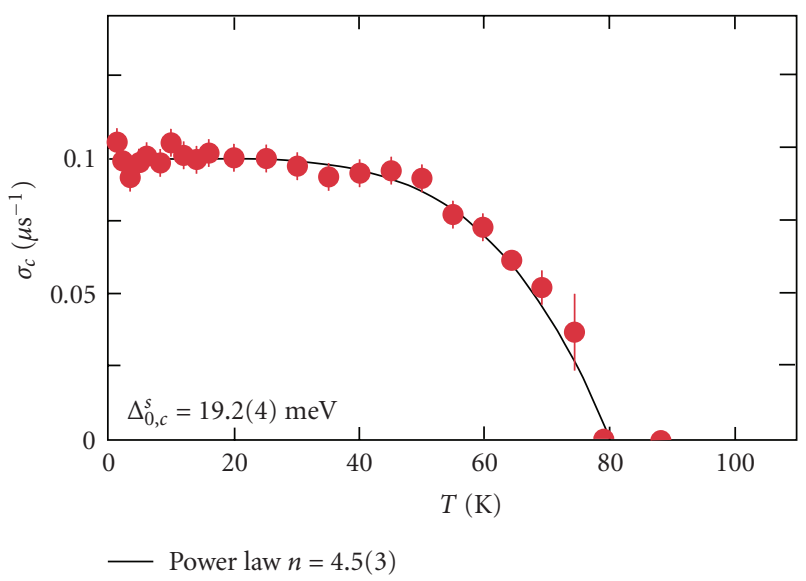

(a)

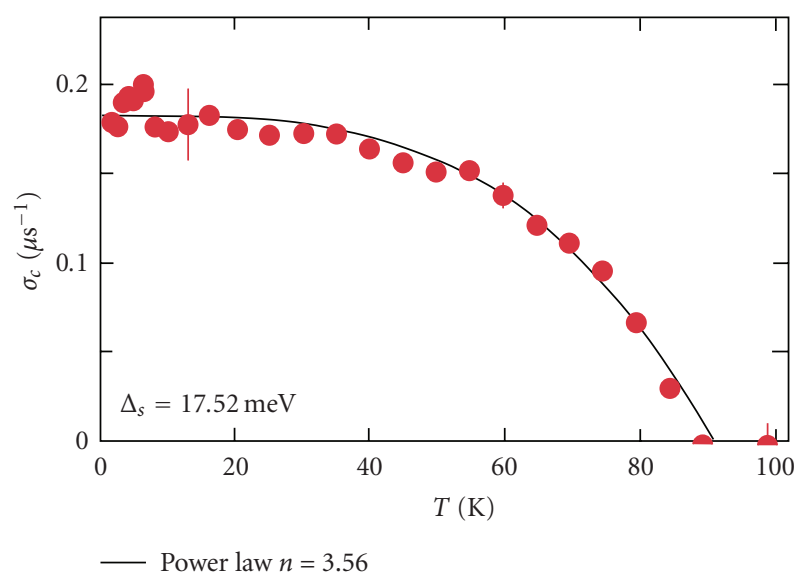

(b)

Figure 10: (a) Temperature dependences of the $\mu$ SR relaxation rate $\sigma_{a} \propto \lambda_{a}^{-2}, \sigma_{b} \propto \lambda_{b}^{-2}$, and $\sigma_{c} \propto \lambda_{c}^{-2}$ of single crystals of $\mathrm{YBa}_{2} \mathrm{Cu}_{4} \mathrm{O}_{8}$ measured along the three principal crystallographic axes $a, b, c$. (b) Temperature dependence of the $\mu \mathrm{SR}$ relaxation rate $\sigma_{a} \propto \lambda_{a}^{-2}, \sigma_{b} \propto \lambda_{b}^{-2}$, and $\sigma_{c} \propto \lambda_{c}^{-2}$ of single crystals of $\mathrm{YBa}_{2} \mathrm{Cu}_{3} \mathrm{O}_{7-\delta}$ measured along the three principal crystallographic axes $a, b, c$. The lines in (a) and (b) are results from model calculations as discussed in [22-25], after [22-25].

addressed above [5-13, 26-28]. This strong interaction between dopant and the lattice leads to local polaron formation. While at high temperatures these polarons are randomly distributed over the lattice, the large strain fields, which accompany them, require a self-organization into dynamical patterns like, for example, stripes [43-46]. This patterning takes place at the onset temperature $T^{*}$ of the pseudogap phase and leads to the coexistence of metallic distorted regions with "pseudo" antiferromagnetic insulating regimes [43-46]. Both regions are not independent of each other but interact dynamically through the lattice and local charge transfer. In the superconducting phase the polarons persist and contribute to superconductivity through pair formation and interband interactions [43-46, 88]. 
Besides of the electronic response to doping and local lattice effects, also the lattice experiences important renormalizations since electronic and lattice degrees of freedom are undistinguishable. This shows up in local mode softening due to the electronic cloud trapped by the lattice and influences-in turn - the relative mean square $\mathrm{Cu}-\mathrm{O}$ lattice displacement [88]. An unusual upturn at the point where the polarons gain coherence appears and another anomaly upon the onset of superconductivity takes place. This second anomaly depends, however, on the pairing symmetry and is most pronounced for the case of an $s$-wave order parameter.

The above described scenario is modeled by the following (simplified) Hamiltonian [43-46, 89, 90]:

$$
H=H_{d}+H_{c}+H_{c d}+H_{L}+H_{L-d}+H_{L-c},
$$

where $H_{d}, H_{c}$ refer to the purely electronic energies of the AFM matrix and the doped holes, and $H_{c d}$ is the interaction between both and resembles a hybridization term. The last three terms are the pure lattice part, the electron-lattice interactions with the AFM holes, and the doped holes, respectively. The terms in (1) are explicitly given by [43$46,88-90]$

$$
\begin{aligned}
H_{d}= & \sum_{i, \sigma} \varepsilon_{d} d_{i, \sigma}^{+} d_{i, \sigma}+\sum_{i, j, \sigma, \sigma^{\prime}} t_{i j}\left(d_{i, \sigma}^{+} d_{j, \sigma^{\prime}}+c . c .\right) \\
& +U \sum_{i} n_{d, i \uparrow} n_{d, i \downarrow}, \\
H_{c}= & \sum_{i, \sigma} \varepsilon_{c} c_{i, \sigma}^{+} c_{i, \sigma}+\sum_{i, j, \sigma, \sigma^{\prime}} t_{i j}\left(c_{i, \sigma}^{+} c_{j, \sigma^{\prime}}+h . c .\right), \\
H_{c d}= & \sum_{i, j, \sigma, \sigma^{\prime}} t_{c d}\left(c_{i, \sigma}^{+} d_{j, \sigma^{\prime}}+c . c .\right), \\
H_{L}= & \sum_{i} \frac{p_{i}^{2}}{2 M_{i}}+\frac{1}{2} M \omega^{2} Q_{i}^{2}, \\
H_{L-d}= & \sum_{i, j, \sigma, \sigma^{\prime}}\left[g n_{d, i} Q_{i}+\tilde{g}\left(c_{i, \sigma}^{+} d_{j, \sigma^{\prime}}+d_{i, \sigma}^{+} c_{j, \sigma^{\prime}}\right) Q_{j}\right], \\
H_{L-c}= & \sum_{i, j, \sigma, \sigma^{\prime}}\left[g n_{c, i} Q_{i}+\tilde{g}\left(c_{i, \sigma}^{+} d_{j, \sigma^{\prime}}+d_{i, \sigma}^{+} c_{j, \sigma^{\prime}}\right) Q_{j}\right] .
\end{aligned}
$$

The electronic states within the antiferromagnetic background are denoted by creation and annihilation operators $d^{+}, d$, with density $n_{d}=d^{+} d$, those within the lattice distorted areas are labelled $c^{+}, c$, and $n_{c}=c^{+} c$. $\varepsilon$ are the site $i$ dependent energies, $t$ are the hopping integrals, and $U$ is the onsite Coulomb repulsion within the AFM matrix. In the hole rich areas $U$ becomes meaningless since it is strongly reduced by the electron-lattice coupling and might even become attractive there. The coupling between the lattice and the electronic subsystems consists of a diagonal coupling proportional to $g$ and an off-diagonal coupling proportional to $\widetilde{g}$ which enables charge transfer processes between the two regimes. Principally, the couplings between the $c$ and $d$ regimes are not identical, but for simplicity they are assumed to be the same here. For simplicity the lattice Hamiltonian is taken to be harmonic with $p$ and $Q$ being site $i$ dependent

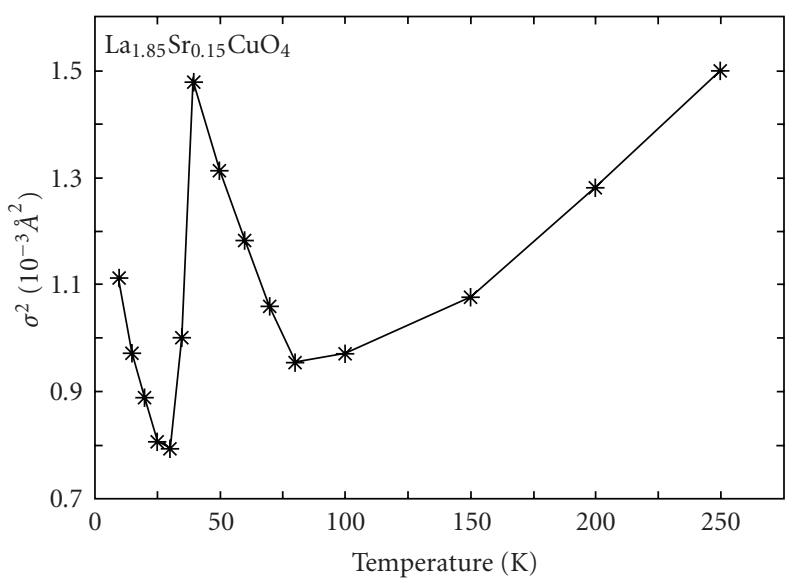

Figure 11: The mean square in-plane $\mathrm{Cu}-\mathrm{O}$ bond distance of $\mathrm{La}_{1.85} \mathrm{Sr}_{0.15} \mathrm{CuO}_{4}$ as a function of temperature. After [26-28].

momentum and conjugate displacement coordinates with frequency $\omega$ and $M$ the ionic mass.

In order to decouple lattice and electronic degrees of freedom a Lang-Firsov canonical transformation [91] is performed which corresponds to $\tilde{H}=e^{-S} H e^{S}$. This induces an exponential band narrowing in the electronic energies together with a level shift proportional to $\Delta^{*}=$ $(1 / 2 N) \sum_{q}\left(g_{q}^{2} / \hbar \omega_{q}\right)$ and a rigid oscillator shift in the lattice degrees of freedom as follows:

$$
\begin{gathered}
\tilde{c}_{i}=c_{i} \exp \left(\sum_{q} g_{q}\left[b_{q}^{+}-b_{q}\right]\right), \\
\tilde{c}_{i}^{+}=c_{i}^{+} \exp \left(-\sum_{q} g_{q}\left[b_{q}^{+}-b_{q}\right]\right), \\
\tilde{d}_{i}=d_{i} \exp \left(\sum_{q} g_{q}\left[b_{q}^{+}-b_{q}\right]\right), \\
\tilde{d}_{i}^{+}=d_{i}^{+} \exp \left(-\sum_{q} g_{q}\left[b_{q}^{+}-b_{q}\right]\right), \\
\tilde{b}_{q}=b_{q}+\sum_{q} g_{q} n_{i}, \quad \tilde{b}_{q}^{+}=b_{q}^{+}+\sum_{q} g_{q} n_{i} .
\end{gathered}
$$

As is obvious from (2), a coupled feedback from the lattice to the electronic degrees of freedom and vice versa results. We first concentrate on the renormalizations of the electronic subsystem and then discuss those experienced by the lattice.

The electronic kinetic energy renormalization is given by $t_{i} \rightarrow \tilde{t}_{i}=t_{i} \exp \left[-g^{2} \operatorname{coth}(\hbar \omega / 2 k T)\right]$, whereas the site dependent energies transform to $\varepsilon \rightarrow \tilde{\varepsilon}-\Delta^{*}, \Delta^{*}=$ $(1 / 2 N) \sum_{q}\left(g_{q}^{2} / \hbar \omega_{q}\right)$, and $U$ has to be replaced by $\tilde{U}=$ $U-\left(g_{q}^{2} / \hbar \omega_{q}\right)$. The lattice-induced hybridization terms experience an important renormalization since density-density interactions are a consequence which facilitate multiband superconductivity and lead to strong enhancements of $T_{c}$ $[92,93]$. 
The electronic part of the transformed Hamiltonian can be cast into an effective BCS scheme which is, however, extended to account for multiband superconductivity. The resulting Hamiltonian reads [43-46, 92, 93]

$$
\begin{aligned}
H & =H_{0}+H_{1}+H_{2}+H_{12}, \\
H_{0}= & \sum_{k_{1} \sigma} \xi_{k_{1}} c_{k_{1} \sigma}^{+} c_{k_{1} \sigma}+\sum_{k_{2} \sigma} \xi_{k_{2}} d_{k_{2} \sigma}^{+} d_{k_{2} \sigma}, \\
H_{1}= & -\sum_{k_{1} k_{1}^{\prime} q} V_{1}\left(k_{1}, k_{1}^{\prime}\right) c_{k_{1}+q / 2 \uparrow}^{+} c_{-k_{1}+q / 2 \downarrow}^{+} c_{-k_{1}^{\prime}+q / 2 \downarrow} c_{k_{1}^{\prime}+q / 2 \uparrow}, \\
H_{2}= & -\sum_{k_{2} k_{2}^{\prime} q} V_{2}\left(k_{2}, k_{2}^{\prime}\right) d_{k_{2}+q / 2 \uparrow}^{+} d_{-k_{2}+q / 2 \downarrow}^{+} d_{-k_{2}^{\prime}+q / 2 \downarrow} d_{k_{2}^{\prime}+q / 2 \uparrow}, \\
H_{12}=- & \sum_{k_{1} k_{2} q} V_{12}\left(k_{1}, k_{2}\right) \\
& \quad \times\left\{c_{k_{1}+q / 2 \uparrow}^{+} c_{-k_{1}+q / 2 \downarrow}^{+} d_{-k_{2}+q / 2 \downarrow} d_{k_{2}+q / 2 \uparrow}+\text { h.c. }\right\},
\end{aligned}
$$

where $H_{0}$ is the kinetic energy of band $c, d$ with $\xi_{k_{i}}=\widetilde{\varepsilon}_{i}-\varepsilon_{k_{i}}-$ $\mu, i=c, d, \mu$ is the chemical potential which controls the band filling, and the band dispersion is given by the simplified scheme as suggested by LDA calculations [94]:

$$
\begin{aligned}
\mathcal{E}_{c, d}(k)= & -2 t_{1}\left(\cos k_{x} a+\cos k_{y} b\right)+4 t_{2} \cos k_{x} a \cos k_{y} b \\
& +2 t_{3}\left(\cos 2 k_{x} a+\cos 2 k_{y} b\right) \\
& \mp \frac{t_{4}\left(\cos k_{x} a-\cos k_{y} b\right)^{2}}{4}-\mu
\end{aligned}
$$

with $t_{1}, t_{2}$, and $t_{3}$ being nearest, next, and third nearest neighbour hopping integrals, whereas $t_{4}$ is the interplanar hopping term which becomes relevant in multilayer systems. Principally, all hopping integrals should be affected by the polaronic effects. It turns out, however, that a renormalization of the 3rd nearest neighbour hopping term has no influence on any of the below investigated physical properties. The terms $H_{1}, H_{2}, H_{12}$ provide the pairing potentials in the $c$ and $d$ bands and the pairwise exchange between both bands. The pairing interactions can be represented in factorized form in order to account for anisotropic pairings like, for example, extended $s$-wave or $d$-wave pairing. Guided by the experimental data which have been presented above (see Figures 9 and 10), it is assumed that in the $c$-channel $s$ wave pairing is realized whereas in the $d$-channel a $d$-wave order parameter exists. From (4) coupled gap equations can be derived which are given by $[92,93]$

$$
\begin{aligned}
\left\langle c_{k_{1}}^{+} c_{-k_{1} \downarrow}^{+}\right\rangle & =\frac{\bar{\Delta}_{k_{1}}}{2 E_{k_{1}}} \tanh \frac{\beta E_{k_{1}}}{2}=\bar{\Delta}_{k_{1}} \Phi_{k_{1}}, \\
\left\langle d_{k_{2}}^{+} d_{-k_{2} \downarrow}^{+}\right\rangle & =\frac{\bar{\Delta}_{k_{2}}}{2 E_{k_{2}}} \tanh \frac{\beta E_{k_{2}}}{2}=\bar{\Delta}_{k_{2}} \Phi_{k_{2}},
\end{aligned}
$$

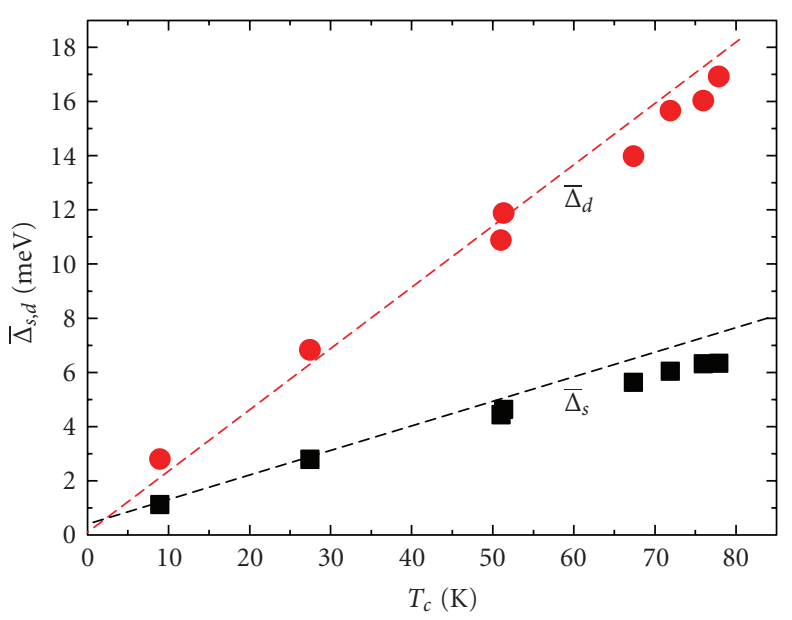

Figure 12: Dependence of the $s$-wave gap $\bar{\Delta}_{s}$ (black squares) and the $d$-wave gap $\bar{\Delta}_{d}$ (red circles) on the corresponding $T_{c}$. The dashed lines are a guide to the eye.

$$
\begin{aligned}
& \bar{\Delta}_{k_{1}}=\sum_{k_{1}^{\prime}} V_{1}\left(k_{1}, k_{1}^{\prime}\right) \bar{\Delta}_{k_{1}^{\prime}} \Phi_{k_{1}^{\prime}}+\sum_{k_{2}} V_{1,2}\left(k_{1}, k_{2}\right) \bar{\Delta}_{k_{2}} \Phi_{k_{2}}, \\
& \bar{\Delta}_{k_{2}}=\sum_{k_{2}^{\prime}} V_{2}\left(k_{2}, k_{2}^{\prime}\right) \bar{\Delta}_{k_{2}^{\prime}} \Phi_{k_{2}^{\prime}}+\sum_{k_{1}} V_{2,1}\left(k_{2}, k_{1}\right) \bar{\Delta}_{k_{1}} \Phi_{k_{1}} .
\end{aligned}
$$

These have to be solved simultaneously and selfconsistently for each temperature $T$, and $T_{c}$ is defined by the condition $\bar{\Delta}_{k_{i}}=0$. The dependencies of these gaps on $T_{c}$ are shown in Figure 12. While the $s$-wave gap to $T_{c}$ ratio is distinctly smaller than the BCS value $\left(2 \bar{\Delta}_{s} / k T_{c}=3.33\right)$, the one of the $d$-wave gap $\left(2 \bar{\Delta}_{d} / k T_{c}=9.68\right)$ is substantially enhanced. The average gap to $T_{c}$ value $\left(2 \bar{\Delta} / k T_{c}=5.34\right)$ remains enhanced as compared to the BCS prediction and agrees with experimentally observed one (see Section 2.1).

Isotope effects on the gaps are possible through the dependence of the band energies on the polaronic coupling which carries a mass dependence. These have been calculated as a function of the chemical potential and are shown in Figure 13. The isotope effects on both gaps are of the same order of magnitude and are doping dependent. In the underdoped regime the OIE is substantially larger than around optimum doping where it almost vanishes (see Figure 6, where the full green line stems from model calculations). Most importantly, however, the model predicts a sign reversal of it in the overdoped regime as is seen experimentally and shown in Figure 6 [17].

A doping dependent OIE on $T_{c}$ stems also from the polaronic renormalization of the single particle energies. Here, however, not all considered hopping integrals contribute equally, but it is found that the nearest neighbour hopping integral $t_{1}$ leads to a sign reversal of the OIE at small dopings and thus can be discarded to contribute to the OIE (see black solid line and symbols in Figure 1). This wrong trend for the isotope effect stemming from the renormalization of $t_{1}$ arises from the related density of states which increases with 


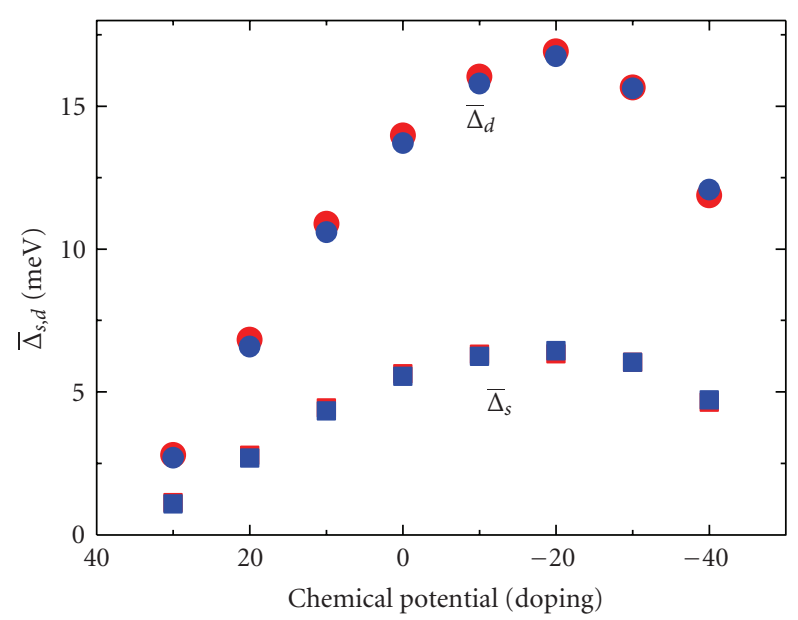

Figure 13: Dependence of the $s$-wave gap $\bar{\Delta}_{s}$ (squares) and $d$-wave gap $\bar{\Delta}_{d}$ (circles) on the chemical potential. The red symbols are for ${ }^{16} \mathrm{O}$, the blue ones for ${ }^{18} \mathrm{O}$.

increasing oxygen ion mass. The correct trend for the OIE is obtained from the renormalization of the second, $t_{2}$, and interplanar hopping integrals, $t_{4}$ (see purple solid line and symbols in Figure 1). This admits to draw conclusions about the relevant lattice mode which causes the OIE, namely, the $Q_{2}$-type Jahn-Teller mode with important contributions from a $c$-axis mode [43-46, 88].

The experimentally observed SOIE (Figures 2 and 3 ) has theoretically been attributed to the density of states at the Fermi energy $E_{F}$ of the related oxygen ions [67]. Since the apical and chain oxygen ions have a small density of states at $E_{F}$ as compared to the in-plane oxygen ions, the large difference in both causes also the difference in their contribution to the total OIE.

From (4)-(6) the superfluid stiffness is calculated within linear response theory [95] through the relation between current and the induced transverse gauge field:

$$
\begin{aligned}
\rho_{s}^{i}= & \frac{1}{2 V} \\
& \times \sum_{k}\left\{\left(\frac{\partial \xi_{k_{i}}}{\partial k_{i}}\right)^{2} \frac{\partial f\left(E_{k_{i}}\right)}{\partial E_{k_{i}}}+\frac{1}{2} \frac{\partial^{2} \xi_{k_{i}}}{\partial k_{i}^{2}}\left[1-\frac{\xi_{k_{i}}}{E_{k_{i}}} \tanh \frac{E_{k_{i}}}{2 k T}\right]\right\},
\end{aligned}
$$

where $E(k)=\sqrt{\xi_{k}^{2}+\bar{\Delta}_{0}^{2}}$. The superfluid stiffness is characterized by two components related to the coupled gaps and has an additional in-plane anisotropy caused by orthorhombicity. Since the two gaps considered above have largely different values (Figures 12 and 13), the superfluid stiffness exhibits an inflection point at low temperatures as is seen experimentally (see Figures 9 and 10). A typical example is shown in Figure 14 which closely resembles the results shown in Figures 9 and 10.

Obviously, also an oxygen isotope dependence on the in-plane penetration depth results from (7). This is in quantitative agreement with the experimental data shown

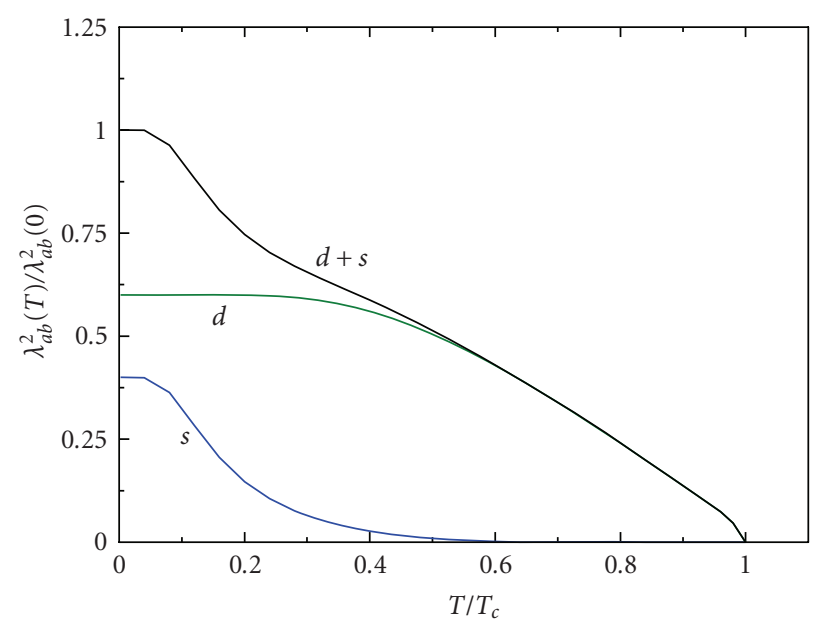

Figure 14: The individual components of $\lambda_{a b}^{2}(T) / \lambda_{a b}^{2}(0)$ as a function of the normalized temperature $T / T_{c}$ (blue: $s$-wave; green: $d$-wave) to the total (black line) normalized in-plane penetration depth.

in Figure 4, as long as the underdoped regime is concerned. The correlation between the OIE on $\lambda_{a b}$ and the one on $T_{c}$ (see Figure 5, solid green line) is, however, only obeyed for small and intermediate doping levels while for optimal doping deviations between experiment and theory are seen. These are-most likely—attributable to the theoretical simplification of using a doping independent polaronic coupling constant $g$ (see (2)).

Finally, the lattice response to polaron formation is addressed. The lattice harmonic oscillators experience a rigid shift proportional to $\sum_{q} g_{q}^{2} n_{i} n_{i}$. By staying at a mean field level and neglecting cross terms, the renormalized phonon frequency is approximated by [88]

$$
\widetilde{\omega}_{q, j}^{2}=\omega_{q, j}^{(0) 2}-\frac{g_{q, j}^{2}}{N\left(E_{F}\right)} \sum_{k} \frac{1}{\varepsilon(k)} \tanh \frac{\varepsilon(k)}{k_{B} T},
$$

where $N\left(E_{F}\right)$ is the density of states at the Fermi level, $\varepsilon(k)$ is given by (5), and $\omega_{q, j}^{(0)}$ is the bare branch $j$ and momentum $q$ dependent lattice mode frequency. For temperatures $T<$ $T_{c} \mathcal{E}(k)$ in (8) has to be replaced by $E(k)=\sqrt{\mathcal{\varepsilon}(k)^{2}+\bar{\Delta}_{0}(k)^{2}}$ with $\bar{\Delta}_{0}(k)$ being the average superconducting energy gap. Its momentum dependence is taken as a parameter: we consider possible $s, d$, and $s+d$ wave symmetries here. From (8), finite momentum mode softening can set in if the normal mode unrenormalized frequency $\omega_{q, j}^{(0)}$ is reduced by the electronic energy, corresponding to $\widetilde{\omega}_{q, j}^{2} \rightarrow 0$. Obviously, this situation is controlled by the dependence on the coupling strength $g_{q, j}$ (8) which we use as a variable to calculate the mode freezing temperature where $\widetilde{\omega}_{q, j}^{2}=0$. Since the electronic energies due to their coupling to the lattice degrees of freedom and the unrenormalized mode energies are both isotope dependent, the freezing temperature is isotope dependent as well. This dependence is huge, sign reversed, and of the same order of magnitude as the experimentally observed OIE on $T^{*}$ (see Figure 8) [19, 74]. In the present approach the freezing 


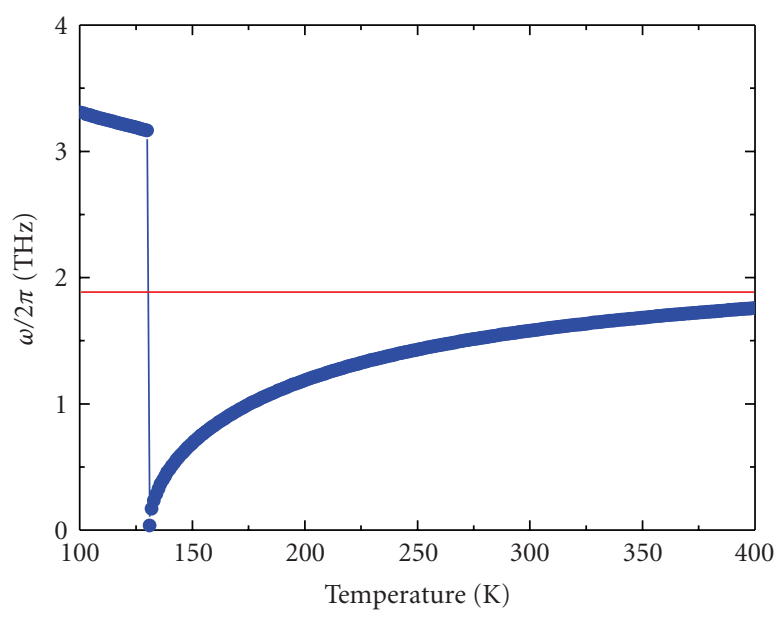

(a)

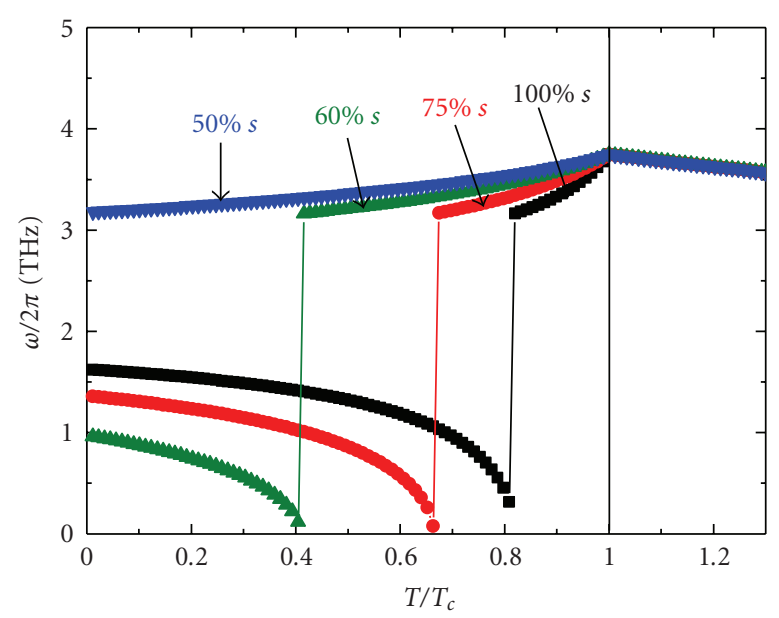

(b)

FIgURE 15: (a) Temperature dependence of the polaronic mode for $T>T_{c}$. The dashed line indicates the unrenormalized local mode frequency and the deviation from it signals the onset of the polaron formation temperature. (b) Dependence of the polaronic mode on the reduced temperature $T / T_{c}$. The colour code refers to the $s+d$ wave admixture as indicated in the graph.

temperature, which we identify with $T^{*}$, signals the onset of a dynamically modulated, patterned structure (of local coherent polarons), where the superstructure modulation is defined by the $q$-value, where $\widetilde{\omega}_{q, j}^{2}=0$ and $T^{*}$ is defined through the implicit relation

$$
\omega_{q, j}^{(o) 2}=\frac{g_{q, j}^{2}}{N\left(E_{F}\right)} \sum_{k} \frac{1}{\varepsilon(k)} \tanh \frac{\varepsilon(k)}{k_{B} T^{*}} .
$$

The complete softening of the renormalized local mode frequency, which signals the onset of polaron coherence, is shown in Figure 15(a) as a function of temperature for $T>$ $T_{c}$. Above $T^{*}$, typical local mode softening is observed beginning approximately $220 \mathrm{~K}$ above $T^{*}$ as is evident from the deviations of the unrenormalized mode frequency indicated by the dashed line in Figure 15(a). This high-temperature scale signals the onset of polaron formation with random distribution on the lattice. At $T^{*}$ the polarons become locally coherent and spatially patterned. Below $T^{*}$ the dynamics are fast but still preserve their spatial self-organization. In this regime almost no temperature dependence in the polaronic mode is present. For temperatures $T<T^{*}$ the opening of the superconducting gap influences these local dynamics massively, since now global coherence sets in. This is especially dramatic if the superconducting order parameter has an $s$-wave component.

In Figure 15(b) this temperature regime is shown with varying amounts of $s$-wave admixture to a $d$-wave order parameter. For a pure $s$-wave order parameter a substantial softening of the polaronic mode is observed in the immediate vicinity of $T_{c}$. With increasing $d$-wave contribution the softening shifts to lower temperatures and is already lost for $50 \% d+50 \% s$ wave composition: only a small cusp at $T_{c}$ indicates the onset of superconductivity.

An important consequence of the above local mode softening is related to the mean square $\mathrm{Cu}-\mathrm{O}$ displacement
$\sigma^{2}(T)$ involved in the polaron formation. Here actually two copper-oxygen displacements are relevant for most HTS materials, one within the $a b$-planes, and the other one referring to the $\mathrm{Cu}$-apical oxygen displacement [1012]. Mostly, however, only the former is accessible to experiments, since this is substantially shorter than the latter one. Using the fluctuation/dissipation theorem we find $\sigma^{2}(T)=\hbar /\left(M \tilde{\omega}_{q, j}\right) \operatorname{coth}\left(\hbar \tilde{\omega}_{q, j} / 2 k_{B} T\right)$. The calculated temperature dependence of $\sigma^{2}(T)$ is shown in Figure 16. Above $T^{*} \sigma^{2}(T)$ obeys the Debye-Waller temperature dependence, however, enhanced at the onset temperature of polaron formation. At $T^{*}$ a divergence in $\sigma^{2}(T)$ appears caused by the freezing of the polaronic mode. Such a behaviour has been observed experimentally in various cuprate HTSs, and a typical experimental result is shown in Figure 11 and in the inset to Figure 16 [5, 7, 8, 10-12, 26-28]. The previously used assignment of $T^{*}$ is different from our definition, since in $[5,7,8,10-12,26-28] T^{*}$ has been related to the onset of deviations from the Debye Waller behaviour; that is, $T^{*}$, in those references, is higher than the actual polaron freezing temperature.

With decreasing coupling strength $g$, not only does $T^{*}$ decrease but also the peak width and height diminish, becoming unobservable for small coupling. For temperatures below and slightly above $T_{c}$ the temperature dependence of $\sigma^{2}(T)$ is strongly affected by the superconducting gap symmetry, as anticipated from the $T$-dependence of the polaronic mode. With increasing admixture of a $d$-wave component to the primarily $s$-wave gap symmetry, the peak in $\sigma^{2}(T)$ shifts to lower temperatures and is completely suppressed if the $d$-wave component achieves $50 \%$ or more. However, a similar suppression of this divergence is found if the renormalization of the hopping elements occurs for the second and interplanar hopping integrals only. In this case the coupling strength is the relevant parameter: the smaller it is, the more the peak is suppressed. The complete 


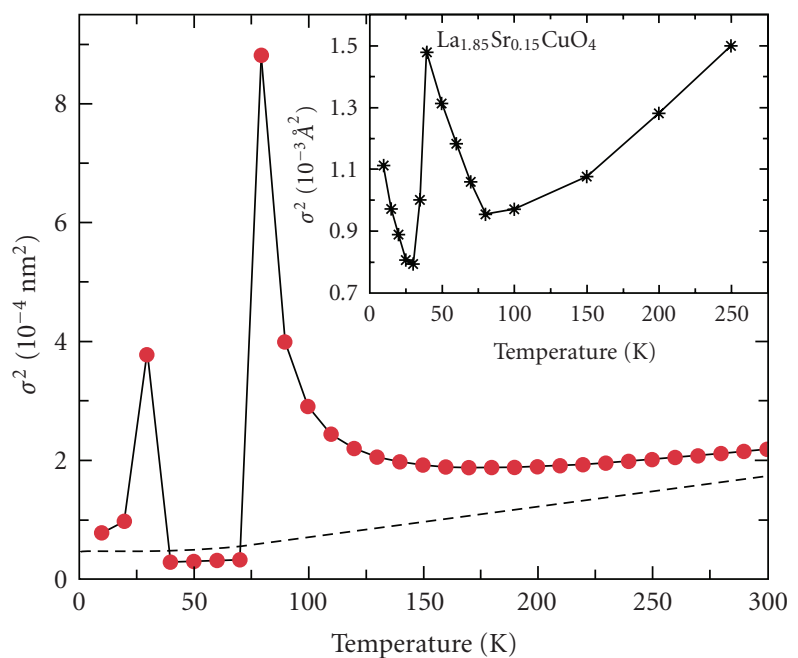

FIGURE 16: Calculated temperature dependence of the mean square $\mathrm{Cu}-\mathrm{O}$ displacement $\sigma^{2}(T) . T^{*}$ is defined as the temperature where the first divergence in $\sigma^{2}(T)$ appears (in this example at $T=100 \mathrm{~K}$ ). The lower temperature divergence occurs at or below $T_{c}$ depending on the pairing symmetry as explained in the text. The inset shows the experimental results obtained in [26-28].

suppression that takes place for a $50 \% s$-wave $+50 \% d$-wave order parameter does not occur, but a substantial shift to temperatures well below $T_{c}$ occurs. This means that whenever a peak in $\sigma^{2}(T)$ is observed for temperatures $T \leq T_{c}$, the superconducting order parameter cannot be purely $d$-wave but must have an additional isotropic s-wave admixture. This conclusion is strongly supported by the above described $\mu \mathrm{SR}$ experiments (see Figures 9 and 10).

\section{Conclusions}

Various unconventional isotope effects observed in cuprate HTSs have been reviewed, and it has been shown that besides of these effects all phases appearing in the complex hole doping dependent phase diagram show isotope dependencies. In addition, compelling experimental evidence has been given that the order parameter in these systems is not a single $d$-wave one but consists of coexisting $s$ - and $d$ wave contributions which change in composition with the crystallographic directions. In the $\mathrm{CuO}_{2}$ planes a well visible $s+d$ wave order parameter is realized whereas along the $c$ axis the $d$-wave part is almost missing and the experimental features are compatible with an $s$-wave order parameter. This finding implies that concentrating on the planes only is rather insufficient and incomplete.

The experimental results have been modelled within a two-component scenario where the doped holes lead to polaron formation and constitute a subsystem in the pseudoAFM matrix. Both components are not phase separated but interact dynamically with each other thereby leading to multiband superconductivity. The observed isotope effects are a natural consequence of polaron formation and convincing agreement with experimental data is achieved.
The local lattice response observed experimentally has been shown to originate from the polaronic feedback effect on the lattice degrees of freedom which results in local mode softening and divergences in the $\mathrm{Cu}-\mathrm{O}$ mean square displacement. Especially at $T^{*}$ the polarons gain coherence and persist in the superconducting phase. Within this scenario the pseudogap phase is a precursor phase to superconductivity which supports it but does not yet reflect the symmetries of the superconducting order parameters. The symmetry of the superconducting order parameter has been shown to influence in a crucial way this displacement, since an $s$-wave order parameter causes another divergence in this displacement whereas a $d$-wave order parameter has almost no effect on it. The experimental data suggest that coupled order parameters are realized.

All experiments described in this review cannot be understood within purely electronic models. In addition, in those the important contributions from the $c$-axis are missing. The data and their theoretical interpretation are in line with the original idea of Jahn-Teller polarons as novel glue for electron pairing, which was the initiating motivation for the discovery of HTSC in cuprates $[1,42]$.

\section{Acknowledgments}

It is a pleasure to acknowledge illuminating discussions with K. A. Müller, H. Oyanagi, A. Simon, R. Khasanov, and A. R. Bishop. Financial support is acknowledged from the Swiss National Science Foundation, the K. Alex Müller Foundation, and the European Program CoMePhS.

\section{References}

[1] J. G. Bednorz and K. A. Müller, "Possible high $T_{c}$ superconductivity in the Ba-La-Cu-O system," Zeitschrift für Physik B, vol. 64, no. 2, pp. 189-193, 1986.

[2] K. A. Müller, in Magnetic Resonance and Relaxation, R. Blinc, Ed., pp. 192-208, North-Holland, Amsterdam, The Netherlands, 1966.

[3] K.-H. Höck, H. Nickisch, and H. Thomas, "Jahn-Teller effect in itinerant electron systems: the Jahn-Teller polaron," Helvetica Physica Acta, vol. 56, pp. 237-243, 1983.

[4] J. P. Franck, in Physical Properties in High Temperature Superconductors IV, D. M. Ginsberg, Ed., pp. 189-293, World Scientific, Singapore, 1994.

[5] A. Bianconi and N. L. Saini, "Nanoscale lattice fluctuations in cuprates and manganites," in Superconductivity in Complex Systems, K. A. Müller and A. Bussmann-Holder, Eds., vol. 114 of Structure and Bonding, pp. 287-330, Springer, Berlin, Germany, 2005.

[6] S. J. L. Billinge, G. H. Kwei, and H. Takagi, "Local octahedral tilts in $\mathrm{La}_{2-x} \mathrm{Ba}_{x} \mathrm{CuO}_{4}$ : evidence for a new structural length scale," Physical Review Letters, vol. 72, no. 14, pp. 2282-2285, 1994.

[7] A. Bianconi, N. L. Saini, A. Lanzara, et al., "Determination of the local lattice distortions in the $\mathrm{CuO}_{2}$ plane of $\mathrm{La}_{1.85} \mathrm{Sr}_{0.15} \mathrm{CuO}_{4}$," Physical Review Letters, vol. 76, no. 18, pp. 3412-3415, 1996.

[8] N. L. Saini, A. Lanzara, H. Oyanagi, et al., "Local lattice instability and stripes in the $\mathrm{CuO}_{2}$ plane of the $\mathrm{La}_{1.85} \mathrm{Sr}_{0.15} \mathrm{CuO}_{4}$ 
system by polarized XANES and EXAFS," Physical Review B, vol. 55, no. 18, pp. 12759-12769, 1997.

[9] R. P. Sharma, S. B. Ogale, Z. H. Zhang, et al., "Phase transitions in the incoherent lattice fluctuations in $\mathrm{YBa}_{2} \mathrm{Cu}_{3} \mathrm{O}_{7-\delta}$," Nature, vol. 404, no. 6779, pp. 736-740, 2000.

[10] J. Mustre de Leon, S. D. Conradson, I. Batistić, and A. R. Bishop, "Evidence for an axial oxygen-centered lattice fluctuation associated with the superconducting transition in $\mathrm{YBa}_{2} \mathrm{Cu}_{3} \mathrm{O}_{7}$," Physical Review Letters, vol. 65, no. 13, pp. 1675$1678,1990$.

[11] A. R. Bishop, D. Mihailovic, and J. Mustre de Leon, "Signatures of mesoscopic Jahn-Teller polaron inhomogeneities in hightemperature superconductors," Journal of Physics: Condensed Matter, vol. 15, no. 9, pp. L169-L175, 2003.

[12] J. Mustre de Leon, M. Acosta-Alejandro, S. D. Conradson, and A. R. Bishop, "Change of the in-plane $\mathrm{Cu}-\mathrm{O}$ bond distribution in $\mathrm{La}_{2} \mathrm{CuO}_{4.1}$ across $T_{c}$," Journal of Physics and Chemistry of Solids, vol. 69, no. 9, pp. 2288-2291, 2008.

[13] T. Egami, "Electron-phonon coupling in high- $T_{c}$ superconductors," in Superconductivity in Complex Systems, K. A. Müller and A. Bussmann-Holder, Eds., vol. 114 of Structure and Bonding, p. 267, Springer, Berlin, Germany, 2005.

[14] H. Uchiyama, A. Q. R. Baron, S. Tsutsui, et al., "Softening of $\mathrm{Cu}-\mathrm{O}$ bond stretching phonons in tetragonal $\mathrm{HgBa}_{2} \mathrm{CuO} \mathrm{O}_{4+\delta}$," Physical Review Letters, vol. 92, no. 19, Article ID 197005, 4 pages, 2004.

[15] T. Fukuda, J. Mizuki, K. Ikeuchi, K. Yamada, A. Q.R. Baron, and S. Tsutsui, "Doping dependence of softening in the bondstretching phonon mode of $\mathrm{La}_{2-x} \mathrm{Sr}_{x} \mathrm{CuO}_{4}(0 \leq x \leq 0.29)$," Physical Review B, vol. 71, no. 6, Article ID 060501, 4 pages, 2005.

[16] H. Keller, "Unconventional isotope effects in cuprate superconductors," in Superconductivity in Complex Systems, K. A. Müller and A. Bussmann-Holder, Eds., vol. 114 of Structure and Bonding, p. 143, Springer, Berlin, Germany, 2005.

[17] R. Khasanov, S. Strässle, K. Conder, E. Pomjakushina, A. Bussmann-Holder, and H. Keller, "Universal correlations of isotope effects in $\mathrm{Y}_{1-x} \mathrm{Pr}_{x} \mathrm{Ba}_{2} \mathrm{Cu}_{3} \mathrm{O}_{7-\delta}$," Physical Review B, vol. 77, no. 10, Article ID 104530, 5 pages, 2008.

[18] R. Khasanov, A. Shengelaya, D. di Castro, et al., "Oxygen isotope effects on the superconducting transition and magnetic states within the phase diagram of $Y_{1-x} \mathrm{PrBa}_{2} \mathrm{Cu}_{3} \mathrm{O}_{7-\delta}$," Physical Review Letters, vol. 101, no. 7, Article ID 077001, 4 pages, 2008.

[19] A. Furrer, "Neutron scattering investigations of charge inhomogeneities," in Superconductivity in Complex Systems, K. A. Müller and A. Bussmann-Holder, Eds., vol. 114 of Structure and Bonding, p. 171, Springer, Berlin, Germany, 2005.

[20] K. A. Müller, "Possible coexistence of s- and d-wave condensates in copper oxide superconductors," Nature, vol. 377, no. 6545, pp. 133-135, 1995.

[21] K. A. Müller and H. Keller, in High $T_{c}$ Superconductivity: 10 years after the Discovery, p. 7, Kluwer Academic Publishers, Dordrecht, The Netherlands, 1997.

[22] R. Khasanov, A. Shengelaya, A. Maisuradze, et al., "Experimental evidence for two gaps in the high-temperature $\mathrm{La}_{1.83} \mathrm{Sr}_{0.17} \mathrm{CuO}_{4}$ superconductor," Physical Review Letters, vol. 98, no. 5, Article ID 057007, 4 pages, 2007.

[23] R. Khasanov, S. Strässle, D. Di Castro, et al., "Multiple gap symmetries for the order parameter of cuprate superconductors from penetration depth measurements," Physical Review Letters, vol. 99, no. 23, Article ID 237601, 4 pages, 2008.

[24] R. Khasanov, A. Shengelaya, J. Karpinski, et al., "s-wave symmetry along the $c$-axis and $s+d$ in-plane superconductivity in bulk $\mathrm{YBa}_{2} \mathrm{Cu}_{4} \mathrm{O}_{8}$," Journal of Superconductivity and Novel Magnetism, vol. 21, no. 2, pp. 81-85, 2008.

[25] A. Bussmann-Holder, R. Khasanov, A. Shengelaya, et al., "Mixed order parameter symmetries in cuprate superconductors," Europhysics Letters, vol. 77, no. 2, Article ID 27002, 4 pages, 2007.

[26] H. Oyanagi, A. Tsukuda, M. Naito, and N. L. Saini, "Local structure of superconducting $(\mathrm{La}, \mathrm{Sr})_{2} \mathrm{CuO}_{4}$ under strain: microscopic mechanism of strain-induced $T_{c}$ variation," Physicsl Review B, vol. 75, no. 2, Article ID 024511, 6 pages, 2007.

[27] H. Oyanagi, C. Zhang, A. Tsukada, and M. Naito, "Lattice instability in high temperature superconducting cuprates probed by X-ray absorption spectroscopy," Journal of Physics: Conference Series, vol. 108, Article ID 012038, 12 pages, 2008.

[28] C. J. Zhang and H. Oyanagi, "Local lattice instability and superconductivity in $\mathrm{La}_{1.85} \mathrm{Sr}_{0.15} \mathrm{Cu}_{1-x} M_{x} \mathrm{O}_{4} \quad(M=$ MN, Ni, and Co)," Physical Review B, vol. 79, no. 6, Article ID 064521, 8 pages, 2009.

[29] S. Robaskiewicz, R. Micnas, and J. Ranninger, "Superconductivity in the generalized periodic Anderson model with strong local attraction," Physical Review B, vol. 36, no. 1, pp. 180-201, 1987.

[30] B. K. Chakraverty, "Superconductive solutions for a two-band Hamiltonian," Physical Review B, vol. 48, no. 6, pp. 4047-4053, 1993.

[31] L. P. Gor'kov and A. V. Sokol, JETP Letters, vol. 46, p. 420, 1987.

[32] W. A. Little and M. J. Holcomb, "Analysis of the pairing interaction in the cuprates," Journal of Superconductivity, vol. 13, no. 5, pp. 695-698, 2000.

[33] C. F. Richardson and N. W. Ashcroft, "Effective electronelectron interactions and the theory of superconductivity," Physical Review B, vol. 55, no. 22, pp. 15130-15145, 1997.

[34] J. E. Hirsch, "Polaronic superconductivity in the absence of electron-hole symmetry," Physical Review B, vol. 47, no. 9, pp. 5351-5358, 1993.

[35] J. B. Goodenough and J.-S. Zhou, "Correlation bag and high$T_{c}$ superconductivity," Physical Review B, vol. 42, no. 7, pp. 4276-4287, 1990.

[36] J. B. Goodenough, J.-S. Zhou, and J. Chan, "Copper oxide superconductors: a distinguishable thermodynamic state," Physical Review B, vol. 47, no. 9, pp. 5257-5286, 1993.

[37] A. S. Alexandrov, "Superconducting polarons and bipolarons," in Polarons in Advanced Materials, A. S. Alexandrov, Ed., Materials Science, p. 257, Springer, Dordrecht, The Netherlands, 2007.

[38] Yu. N. Ovchinnikov, S. A. Wolf, and V. Z. Kresin, "Inhomogeneity of the superconducting state and consequent diamagnetism above $T_{c}$ : application to the cuprates," Physical Review B, vol. 60, no. 6, pp. 4329-4333, 1999.

[39] Yu. N. Ovchinnikov, S. A. Wolf, and V. Z. Kresin, "Intrinsic inhomogeneities in superconductors and the pseudogap phenomenon," Physical Review B, vol. 63, no. 6, Article ID 064524, 6 pages, 2001.

[40] Yu. N. Ovchinnikov and V. Z. Kresin, "Inhomogeneous superconductor in an ac field: application to the pseudogap region," Physical Review B, vol. 65, no. 21, Article ID 214507, 8 pages, 2002.

[41] H. Kamimura, S. Matsuno, Y. Suwa, and H. Ushio, "Occurrence of d-wave pairing in the phonon-mediated mechanism of high temperature superconductivity in cuprates," Physical Review Letters, vol. 77, no. 4, pp. 723-726, 1996. 
[42] K. A. Müller, "On the superconductivity in hole doped cuprates," Journal of Physics: Condensed Matter, vol. 19, no. 25, Article ID 251002, 13 pages, 2007.

[43] A. Bussmann-Holder and H. Keller, "Polaron formation as origin of unconventional isotope effects in cuprate superconductors," European Physical Journal B, vol. 44, pp. 487-490, 2005.

[44] A. Bussmann-Holder, H. Keller, and K. A. Müller, "Evidences for poaron formation in cuprates," in Superconductivity in Complex Systems, K. A. Müller and A. Bussmann-Holder, Eds., vol. 114 of Structure and Bonding, p. 367, Springer, Dordrecht, The Netherlands, 2005.

[45] A. Bussmann-Holder and H. Keller, "Polaron effects in high- $T_{c}$ cuprate superconductors," in Polarons in Advanced Materials, A. S. Alexandrov, Ed., Materials Science, p. 599, Springer, Dordrecht, The Netherlands, 2007.

[46] H. Keller, A. Bussmann-Holder, and K. A. Müller, "Jahn-Teller physics and high- $T_{c}$ superconductivity," Materials Today, vol. 11 , pp. 38-46, 2008.

[47] H. Suhl, B. T. Matthias, and L. Walker, "Bardeen-cooperschrieffer theory of superconductivity in the case of overlapping bands," Physical Review Letters, vol. 3, no. 12, pp. 552$554,1959$.

[48] V. Moskalenko, "Superconductivity of metals taking into account overlapping of the energy bands," Fiz. Metal. Metalov., vol. 8, p. 503, 1959.

[49] B. Geilikman, R. Zaizsev, and V. Z. Kresin, Fiz. Tverd. Tela., vol. 9, p. 693, 1967.

[50] B. Geilikman, R. Zaizsev, and V. Z. Kresin, Soviet Physics-Solid State, vol. 9, p. 542, 1966.

[51] V. Z. Kresin and S. Wolf, "Multigap structure in the cuprates," Physica C, vol. 169, no. 5-6, pp. 476-484, 1990.

[52] V. Z. Kresin and S. A. Wolf, "Major normal and superconducting parameters of high- $T_{c}$ oxides," Physical Review B, vol. 41, no. 7, pp. 4278-4285, 1990.

[53] V. Z. Kresin and S. A. Wolf, "Induced superconducting state and two-gap structure: application to cuprate superconductors and conventional multilayers," Physical Review B, vol. 46, no. 10, pp. 6458-6471, 1992.

[54] V. Z. Kresin, S. Wolf, and G. Deutscher, "The effect of phononmediated charge transfer and internal proximity effect on the properties of multigap cuprate superconductors," Physica C, vol. 191, no. 1-2, pp. 9-14, 1992.

[55] D. Zech, H. Keller, K. Conder, et al., "Site-selective oxygen isotope effect in optimally doped $\mathrm{YBa}_{2} \mathrm{Cu}_{3} \mathrm{O}_{6+x}$, Nature, vol. 371, no. 6499, pp. 681-683, 1994.

[56] G.-M. Zhao, K. Conder, H. Keller, and K. A. Müller, "Oxygen isotope effects in $\mathrm{La}_{2-x} \mathrm{Sr}_{x} \mathrm{CuO}_{4}$ : evidence for polaronic charge carriers and their condensation," Journal of Physics: Condensed Matter, vol. 10, no. 40, pp. 9055-9066, 1998.

[57] G. M. Zhao, H. Keller, and K. Conder, "Unconventional isotope effects in the high-temperature cuprate superconductors," Journal of Physics: Condensed Matter, vol. 13, no. 29, pp. R569-R587, 2001.

[58] K. A. Müller, "On the oxygen isotope effect and apex anharmonicity in high $T_{c}$ cuprates," Zeitschrift für Physik B, vol. 80, p. 193, 1990.

[59] A. Bussmann-Holder and A. R. Bishop, "Anharmonicity in the $c$ direction of high- $T_{c}$ oxides," Physical Review B, vol. 51, no. 10, pp. 6640-6644, 1995.

[60] A. Bussmann-Holder and A. R. Bishop, "Anharmonic electron and phonon effects in the $c$-axis infrared conductivity of high$T_{c}$ oxides," Physical Review B, vol. 51, no. 5, pp. 3246-3249, 1995.
[61] A. Bussmann-Holder and A. R. Bishop, "Anharmonicityinduced multiphonon processes in high-temperature superconductors," Physical Review B, vol. 44, no. 6, pp. 2853-2856, 1991.

[62] A. Bussmann-Holder, A. R. Bishop, and I. Batistić, "Unconventional electron-phonon interactions in high-temperature superconductors," Physical Review B, vol. 43, no. 16, pp. 13728-13731, 1991.

[63] D. Mihailović, I. Poberaj, and A. Mertelj, "Characterization of the pyroelectric effect in $\mathrm{YBa}_{2} \mathrm{Cu}_{3} \mathrm{O}_{7-\delta}$," Physical Review B, vol. 48, no. 22, pp. 16634-16640, 1993.

[64] M. Cardona, R. Liu, C. Thomsen, et al., "Effect of isotopic substitution of oxygen on $T_{c}$ and the phonon frequencies of high $T_{c}$ superconductors," Solid State Communications, vol. 67, no. 8, pp. 789-793, 1988.

[65] G.-M. Zhao, J. W. Ager III, and D. E. Morris, "Site dependence of large oxygen isotope effect in $\mathrm{Y}_{0.7} \mathrm{Pr}_{0.3} \mathrm{Ba}_{2} \mathrm{Cu}_{3} \mathrm{O}_{6.97}$," Physical Review B, vol. 54, no. 21, pp. 14982-14985, 1996.

[66] R. Khasanov, A. Shengelaya, E. Morenzoni, et al., "Siteselective oxygen isotope effect on the magnetic-field penetration depth in underdoped $\mathrm{Y}_{0.6} \mathrm{Pr}_{0.4} \mathrm{Ba}_{2} \mathrm{Cu}_{3} \mathrm{O}_{7-\delta}$," Physical Review B, vol. 68, no. 22, Article ID 220506, 4 pages, 2003.

[67] A. Bussmann-Holder, A. R. Bishop, L. Genzel, and A. Simon, "Doping dependence of $T_{c}$ and its related isotope effect in high-temperature superconductors," Physical Review B, vol. 55, no. 17, pp. 11751-11755, 1997.

[68] J. Hofer, K. Conder, T. Sasagawa, et al., "Oxygen-isotope effect on the in-plane penetration depth in underdoped $\mathrm{La}_{2-x} \mathrm{Sr}_{x} \mathrm{CuO}_{4}$ single crystals," Physical Review Letters, vol. 84, no. 18, pp. 4192-4195, 2000.

[69] A. Bussmann-Holder, H. Keller, A. R. Bishop, A. Simon, R. Micnas, and K. A. Müller, "Unconventional isotope effects as evidence for polaron formation in cuprates," Europhysics Letters, vol. 72, no. 3, pp. 423-429, 2005.

[70] G.-M. Zhao and D. E. Morris, "Observation of a possible oxygen isotope effect on the effective mass of carriers in $\mathrm{YBa}_{2} \mathrm{Cu}_{3} \mathrm{O}_{6.94}$," Physical Review B, vol. 51, no. 22, pp. 1648716490, 1995.

[71] G.-M. Zhao, M. B. Hunt, H. Keller, and K. A. Müller, "Evidence for polaronic supercarriers in the copper oxide superconductors $\mathrm{La}_{2-x} \mathrm{Sr}_{x} \mathrm{CuO}_{4}$," Nature, vol. 385, no. 6613, pp. 236-238, 1997.

[72] R. Khasanov, D. G. Eshchenko, H. Luethkens, et al., "Direct observation of the oxygen isotope effect on the inplane magnetic field penetration depth in optimally doped $\mathrm{YBa}_{2} \mathrm{Cu}_{3} \mathrm{O}_{7-\delta}$," Physical Review Letters, vol. 92, no. 5, Article ID 057602, 4 pages, 2004.

[73] A. Shengelaya, G.-M. Zhao, C. M. Aegerter, K. Conder, I. M. Savic, and H. Keller, "Giant oxygen isotope effect on the spin glass transition in $\mathrm{La}_{2-x} \mathrm{Sr}_{x} \mathrm{Cu}_{1-z} \mathrm{Mn}_{z} \mathrm{O}_{4}$ as revealed by muon spin rotation," Physical Review Letters, vol. 83, no. 24, pp. 5142-5145, 1999.

[74] A. Lanzara, G. M. Zhao, N. L. Saini, et al., "Oxygenisotope shift of the charge-stripe ordering temperature in $\mathrm{La}_{2-x} \mathrm{Sr}_{x} \mathrm{CuO}_{4}$ from X-ray absorption spectroscopy," Journal of Physics: Condensed Matter, vol. 11, pp. L541-L546, 1999.

[75] F. Raffa, T. Ohno, M. Mali, et al., "Isotope dependence of the spin gap in $\mathrm{YBa}_{2} \mathrm{CU}_{4} \mathrm{O}_{8}$ as determined by $\mathrm{Cu}$ NQR relaxation," Physical Review Letters, vol. 81, no. 26, pp. 5912-5915, 1998.

[76] C. C. Tsuei and J. R. Kirtley, "Pairing symmetry in cuprate superconductors," Reviews of Modern Physics, vol. 72, no. 4, pp. 969-1016, 2000.

[77] J. A. Martindale, P. C. Hammel, W. L. Hults, and J. L. Smith, "Temperature dependence of the anisotropy of the planar 
oxygen nuclear spin-lattice relaxation rate in $\mathrm{YBa}_{2} \mathrm{Cu}_{3} \mathrm{O}_{y}$," Physical Review B, vol. 57, no. 18, pp. 11769-11774, 1998.

[78] J. Haase and C. P. Slichter, "Charge density variations or stripes in $\mathrm{YBa}_{2} \mathrm{Cu}_{3} \mathrm{O}_{6+y}$," Journal of Superconductivity, vol. 1, no. 3, pp. 473-475, 2003.

[79] B. Friedl, C. Thomsen, and M. Cardona, "Determination of the superconducting gap in $R \mathrm{Ba}_{2} \mathrm{Cu}_{3} \mathrm{O}_{7-\delta}$," Physical Review Letters, vol. 65, no. 7, pp. 915-918, 1990.

[80] T. Masui, M. Limonov, H. Uchiyama, S. Lee, and S. Tajima, "Raman study of carrier-overdoping effects on the gap in high$T_{c}$ superconducting cuprates," Physical Review B, vol. 68, no. 6, Article ID 060506, 4 pages, 2003.

[81] J. R. Kirtley, C. C. Tsuei, A. Ariando, C. J. M. Verwijs, S. Harkema, and H. Hilgenkamp, "Angle-resolved phasesensitive determination of the in-plane gap symmetry in $\mathrm{YBa}_{2} \mathrm{CU}_{3} \mathrm{O}_{7_{-\delta}}$," Nature Physics, vol. 2, no. 3, pp. 190-194, 2006.

[82] A. Furrer, "Admixture of an s-wave component to the d-wave gap symmetry in high-temperature superconductors," Journal of Superconductivity and Novel Magnetism, vol. 21, no. 1, pp. $1-5,2008$.

[83] A. G. Sun, D. A. Gajewski, M. B. Maple, and R. C. Dynes, "Observation of Josephson pair tunneling between a high- $T_{c}$ cuprate $\left(\mathrm{YBa}_{2} \mathrm{Cu}_{3} \mathrm{O}_{7-\delta}\right)$ and a conventional superconductor (Pb)," Physical Review Letters, vol. 72, no. 14, pp. 2267-2270, 1994.

[84] Q. Li, Y. N. Tsay, M. Suenaga, R. A. Klemm, G. D. Gu, and N. Koshizuka, " $\mathrm{Bi}_{2} \mathrm{Sr}_{2} \mathrm{CaCu}_{2} \mathrm{O}_{8+\delta}$ bicrystal $c$-axis twist josephson junctions: a new phase-sensitive test of order parameter symmetry," Physical Review Letters, vol. 83, no. 20, pp. 41604163, 1999.

[85] G. Binnig, A. Baratoff, H. E. Hoenig, and J. G. Bednorz, "Two-band superconductivity in $\mathrm{Nb}$-Doped $\mathrm{SrTiO}_{3}$," Physical Review Letters, vol. 45, no. 16, pp. 1352-1355, 1980.

[86] F. Giubileo, D. Roditchev, W. Sacks, et al., "Two-gap state density in $M g B_{2}$ : a true bulk property or a proximity effect?" Physical Review Letters, vol. 87, no. 17, Article ID 177008, 4 pages, 2001.

[87] R. Khasanov, D. V. Evtushinsky, A. Amato, et al., "Two-gap superconductivity in $\mathrm{Ba}_{1-x} \mathrm{~K}_{x} \mathrm{Fe}_{2} \mathrm{As}_{2}$ : a complementary study of the magnetic penetration depth by muon-spin rotation and angle-resolved photoemission," Physical Review Letters, vol. 102, no. 18, Article ID 187005, 4 pages, 2009.

[88] A. Bussmann-Holder, H. Keller, A. R. Bishop, A. Simon, and K. A. Müller, "Polaron coherence as origin of the pseudogap phase in high temperature superconducting cuprates," Journal of Superconductivity and Novel Magnetism, vol. 21, no. 6, pp. 353-357, 2008.

[89] A. Bussmann-Holder and H. Keller, "Evidence for polaron formation in high-temperature superconducting cuprates: experiment and theory," Journal of Superconductivity and Novel Magnetism, vol. 22, no. 2, pp. 123-129, 2009.

[90] A. Bussmann-Holder and H. Keller, "Unconventional isotope effects, multi-component superconductivity and polaron formation in high temperature cuprate superconductors," Journal of Physics: Conference Series, vol. 108, Article ID 012019, 16 pages, 2008.

[91] S. G. Lang and Yu. A. Firsov, "Kinetic theory of semiconductors with low mobility," Soviet Physics-JETP, vol. 16, p. 1302, 1963.

[92] A. Bussmann-Holder, R. Micnas, and A. R. Bishop, "Enhancements of the superconducting transition temperature within the two-band model," European Physical Journal B, vol. 37, no. 3, pp. 345-348, 2003.
[93] R. Micnas, S. Robaskiewicz, and A. Bussmann-Holder, "Twocomponent scenario for non-conventional (exotic) superconductors," in Superconductivity in Complex Systems, K. A. Müller and A. Bussmann-Holder, Eds., vol. 114 of Structure and Bonding, p. 13, Springer, Dordrecht, The Netherlands, 2005.

[94] E. Pavarini, I. Dasgupta, T. Saha-Dasgupta, O. Jepsen, and O. K. Anderson, "Band-structure trend in hole-doped cuprates and correlation with $T_{c}$," Physical Review Letters, vol. 87, no. 4, Article ID 047003, 4 pages, 2001.

[95] R. Micnas and B. Tobijaszewska, "Superfluid properties of the extended Hubbard model with intersite electron pairing," Journal of Physics: Condensed Matter, vol. 14, no. 41, pp. 96319649, 2002. 

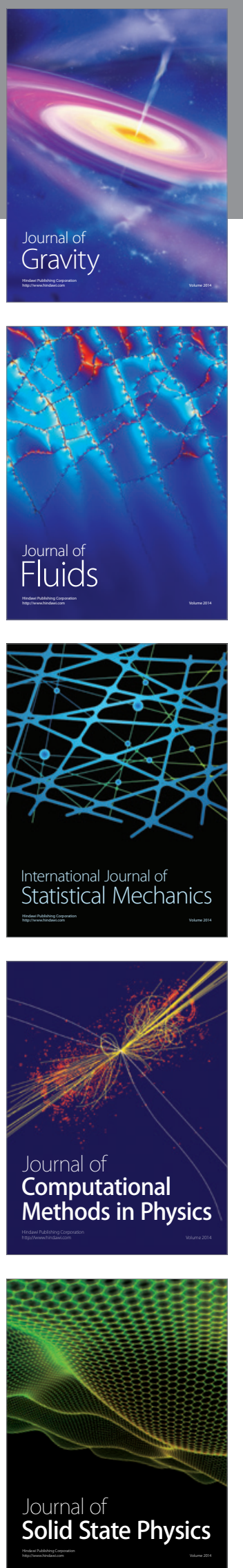

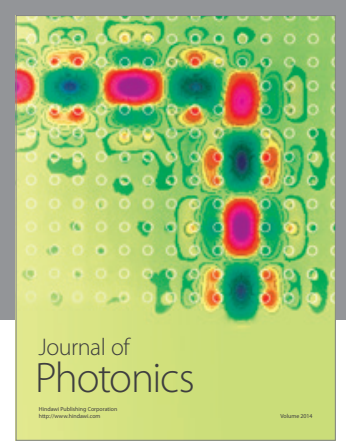

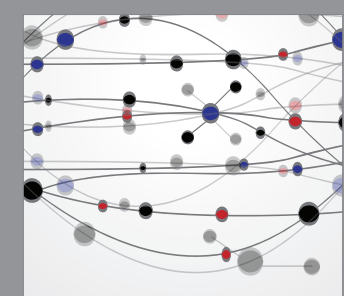

The Scientific World Journal
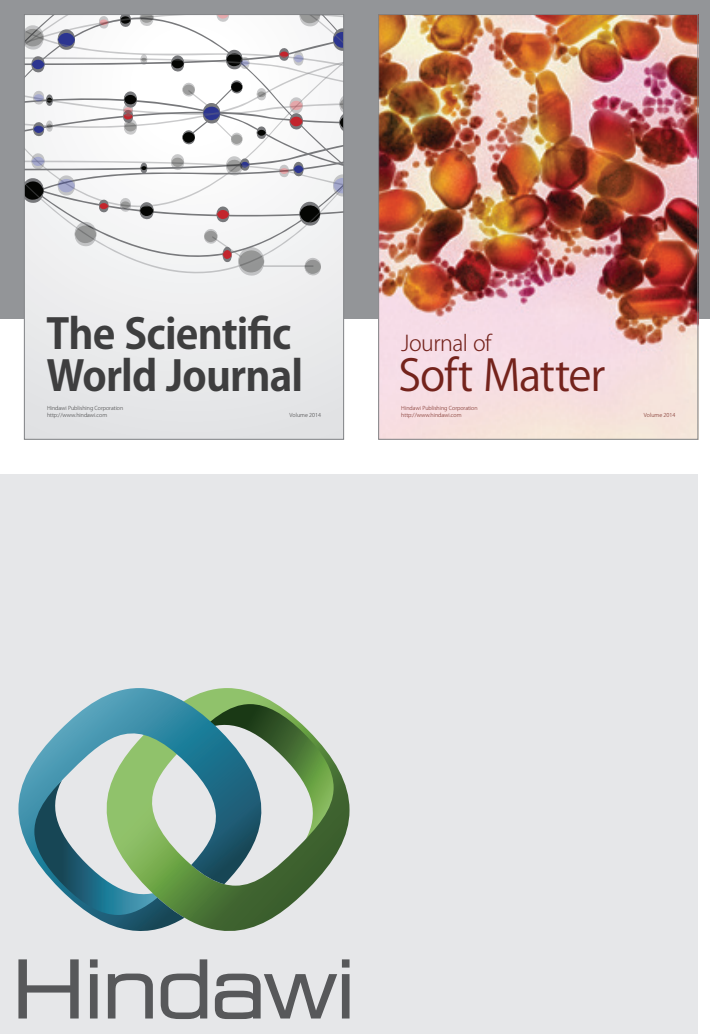

Submit your manuscripts at

http://www.hindawi.com
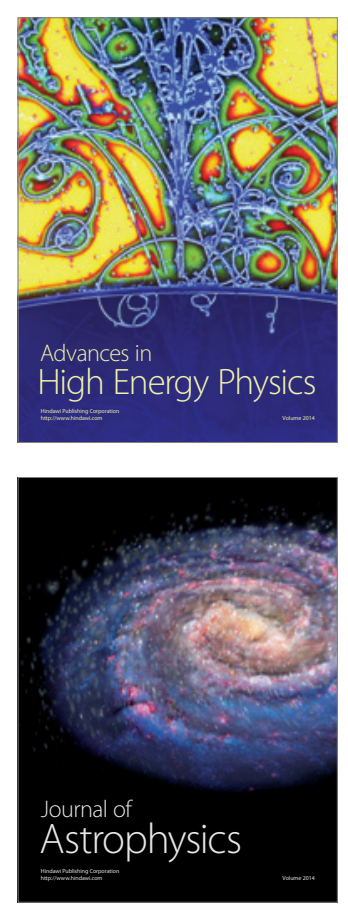
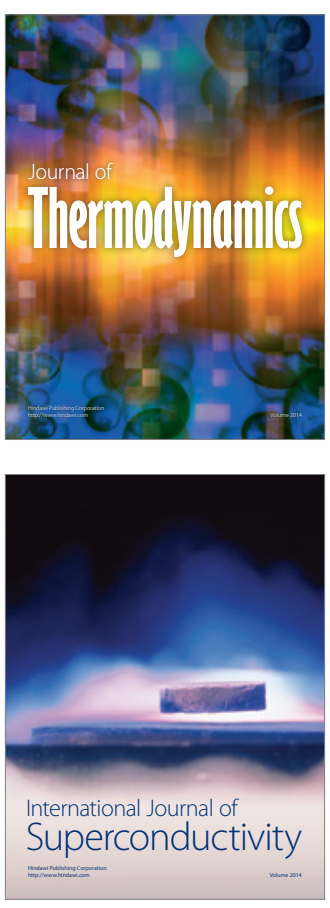
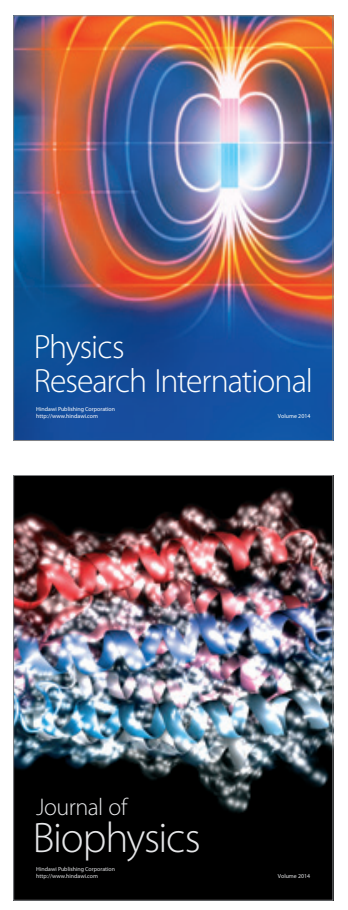
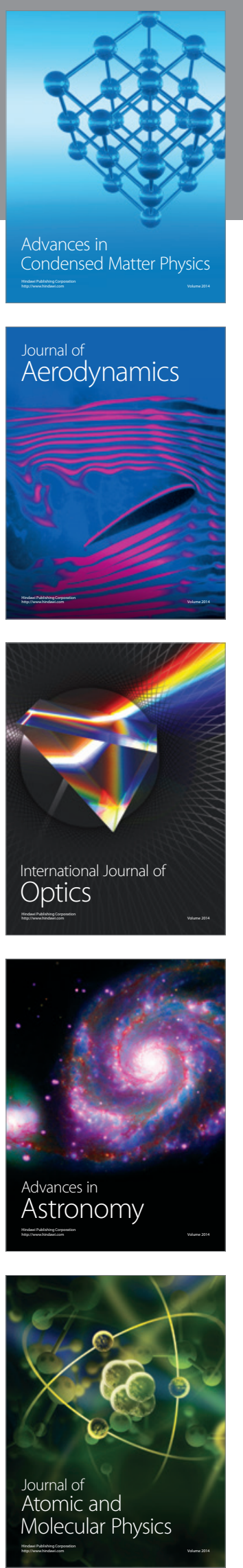